Check for updates

Cite this: Mater. Chem. Front. 2021, 5, 3322

Received 30th December 2020 Accepted 26th February 2021

DOI: 10.1039/d0qm01132k

rsc.li/frontiers-materials

\section{Materials with aggregation-induced emission characteristics for applications in diagnosis, theragnosis, disease mechanism study and personalized medicine}

\begin{abstract}
Engui Zhao (iD ${ }^{a}$ and Sijie Chen (D)*b
Investigations on healthcare-related subjects are becoming increasingly popular among scientists, focusing on the early diagnosis of diseases, innovations in disease treatments and gaining mechanistic insights into disease occurrence, development and recovery. Fluorescent materials with aggregation-induced emission (AIE) characteristics are widely employed in healthcare-related research. By taking advantage of their strong solid-state emission, excellent photostability, fast and sensitive fluorescence responses, and good biocompatibility, materials with AIE attributes are widely employed in pre-clinical diagnosis and mechanistic studies on biological processes. Besides, some AlE-active materials also demonstrate the ability to sensitize the generation of reactive oxygen species (ROS), and have been utilized in photodynamic therapy (PDT). With fluorescence response, AlE-active photosensitizers could light up the targets and exert therapeutic effects at the same time, which enables the theragnosis of infections and tumours. In this review, we select some representative examples of fluorescent materials with AIE attributes and introduce their applications in healthcare-related areas, aiming to illustrate the recent advances in pre-clinical diagnosis, theragnosis, disease mechanism studies and personalized medicines. Since investigations on AIE materials are developing rapidly, we could not cover all reference papers in this review. This review is expected to provide a general idea about the recent progress in this area, rather than a comprehensive encyclopedia to cover all related works.
\end{abstract}

${ }^{a}$ School of Science, Harbin Institute of Technology, Shenzhen, HIT Campus of University Town, Shenzhen 518055, China

${ }^{b}$ Ming Wai Lau Centre for Reparative Medicine, Karolinska Institutet, Hong Kong, China. E-mail: sijie.chen@ki.se

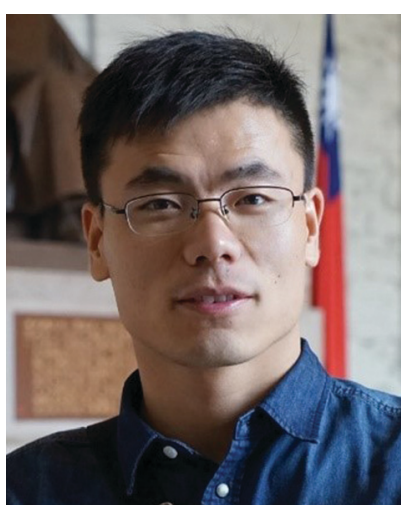

Engui Zhao
Engui Zhao obtained his PhD from the Hong Kong University of Science and Technology in 2015. Afterwards, he worked as a postdoctoral fellow at the same university. In 2016, he joined the Dow Chemical Company in Hong Kong as a Senior Scientist. In 2018, he was recruited by the Dongguan University of Technology as an Assistant Professor. Since 2020, he has been at the Harbin Institute of Technology (Shen Zhen) as an Assistant Professor. His research focus is on the development of fluorescent materials and the exploration of their applications in biomedical fields.

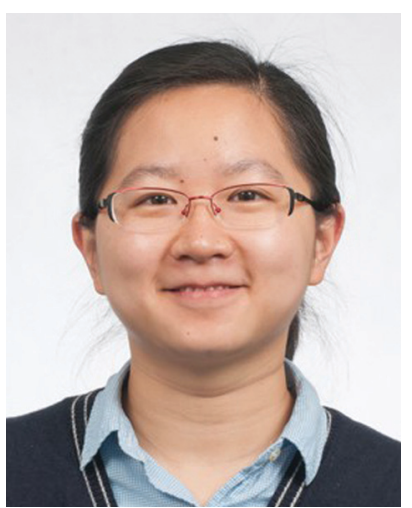

Sijie Chen
Sijie Chen obtained her PhD from the Hong Kong University of Science and Technology in 2013. She received the Endeavour Fellowship and worked as an Endeavour Fellow at the University of Melbourne and as a visiting scientist at the Walter and Eliza Hall Institute of Medical Research (WEHI) in 2015. Thereafter, she joined Karolinska Institutet (KI) in Sweden as a postdoctoral fellow. Dr Chen was recruited as an Assistant Professor at Ming Wai Lau Centre for Reparative Medicine, KI, Hong Kong in 2017. Her current research interests focus on the development of new luminescent materials and techniques for biological research. 


\section{Introduction}

As the standard of living improves, increasing attention is being drawn to healthcare. ${ }^{1}$ Apart from longevity, people are in intense pursuit of the quality of life. Disease is a lion in the way for quality-of-life and may sometimes threaten the human life span. To fight against diseases, early diagnosis, proper medical treatments and understanding of disease mechanisms or medicine functioning mechanisms are excellent weapons for humans. The former two weapons help to conquer diseases by identifying them at an early stage and curing them before they exert significant or irreversible effects on humans, while the third weapon contributes to the development of effective medicines or innovations in treatment methods. The development of all three areas is also the research focus of scientists. Scientists are always fascinated by novel diagnosis methods, better disease treatments, as well as a comprehensive understanding of the disease working mechanisms and how treatments contribute to the cure of certain diseases. With the great research endeavour by scientists worldwide, great progress has been made to advance healthcare.

Fluorescence-based techniques with high sensitivity and selectivity, visible results, ease of application and non-invasiveness, are becoming increasingly popular in healthcare-related research. ${ }^{2-4}$ However, most conventional fluorophores suffer from selfquenching at high concentrations or in the solid-state. The phenomenon was termed as aggregation-caused quenching (ACQ), which was first reported by Förster and Kasper. ${ }^{5,6}$ Since then, the phenomenon has been well studied and its mechanism has been revealed. Traditional fluorophores, such as pyrene and fluorescein, are usually disk-shaped with rigid and coplanar structures, making them excellent emitters in dilute solutions. ${ }^{7,8}$ At high concentrations or in the solid-state, these disk-shaped emitters will experience strong $\pi-\pi$ interactions, and the excitedstate energy will decay through non-radiative photophysical pathways. The ACQ effect or concentration quenching effect of conventional fluorophores makes them unsuitable for applications at high concentrations or as solid particles.

In 2001, Tang, et al. reported a phenomenon that was the opposite of the ACQ effect. ${ }^{7-9}$ A species of propeller-shaped fluorophores emitted dimly in the solution-state but became strong emitters upon forming aggregates. They termed the phenomenon as aggregation-induced emission (AIE). Since then, systematic investigations have been conducted and the mechanistic pictures of this photophysical process have been depicted as follows: AIE luminogens (AIEgens) with propellershaped structures can undergo dynamic rotational or vibrational motions in solution. Such motions can consume the excited-state energy of the fluorophores non-radiatively. In the solid-state or as particles, AIEgens are in close vicinity and the impedance from adjacent molecules restricts the dynamic motions. Besides, the propeller shape of AIE fluorophores is unfavourable for strong $\pi-\pi$ interactions, which excludes the possibility of forming excimers or exciplexes. The rigid molecular conformation without strong $\pi-\pi$ interactions enables AIEgens to decay their excited-state energy through radiative channels, thus AIEgens fluoresce intensely in the aggregated state. Compared with ACQ fluorophores, AIEgens are more suitable for working at high concentrations or in the aggregated states, which is advantageous for practical applications. The strong solid-state emission of AIEgens makes them excellent candidates for solid-state applications, while the fluorescence responses of AIEgens upon forming aggregates or disaggregation facilitate the construction of variable sensing systems based on AIEgens. As such, lots of attempts have been made to explore their practical applications in sensing, ${ }^{10-12}$ imaging, ${ }^{13-21}$ displays $^{22-25}$ and smart materials. ${ }^{26-30}$

Intriguingly, some AIEgens demonstrate the ability to sensitize the formation of reactive oxygen species (ROS) upon light irradiation. ${ }^{31-39}$ ROS can destroy biomolecules and kill cancer cells or microorganisms, realizing photodynamic therapy (PDT). The effectiveness of PDT is positively associated with the ROS sensitizing efficiency of the photosensitizers (PSs) employed. Most traditional PSs with rigid and coplanar structures will undergo strong intermolecular $\pi-\pi$ interactions, and suffer from diminished ROS sensitizing efficiency, making them unsuitable for applications as nanoparticles or at high concentrations. This is detrimental for PDT applications, which usually involves longterm and repeated light irradiation of PSs to produce enough ROS, and requires PSs with good photostability. Research efforts have been invested to avoiding the close interactions of these PSs, such as introducing bulky groups to migrate chromophore aggregation ${ }^{40-44}$ and using supramolecular approaches to separate chromophores, ${ }^{45}$ aiming at increasing the ROS sensitizing efficiency. This usually involves tedious synthetic procedures and time-consuming purifications, which raise the costs of preparation. In contrast, AIE-active molecules not only emit more efficiently in the aggregated state, but also show higher ROS sensitizing efficiency, which makes them excellent candidates for PDT applications. ${ }^{46,47}$ As such, investigations on using AIEtype PSs for PDT are becoming increasingly popular, with plenty of reference papers being published every year.

In this review, we have selected some representative examples to illustrate the applications of AIEgens in healthcare-related research fields and how AIEgens are contributing to the associated technological innovations. Specifically, we will focus on the recent progress on employing AIEgens in diagnosis, theragnosis and their applications in deciphering biological processes and providing guidance for personalized medicine.

\section{Diagnosis}

Diagnosis is the identification of disease causes and nature by a series of patients' symptoms or parameters. As diagnosis determines the subsequent treatment that may be adopted, misdiagnosis may lead to the wrong treatment and result in missing the best opportunity for recovery. Another important aspect of diagnosis is to detect disease before it results in any symptoms. Some diseases, such as pancreatic cancer and colorectal cancer, may not arouse any discomfort in the early stages, but once symptoms appear, they have already become malignant. ${ }^{48}$ Thus, the early and precise detection of disease may greatly benefit patients. For a long time, scientists have been devoting efforts to 
the development of fast, reliable and cost-effective diagnosis methods, among which fluorescent probes are widely employed. AIEgens are sensitive to the changes in microenvironments, such as aggregation/disaggregation, binding to/dissociate from macromolecules, attachment/removal of fluorescence quenchers, structure rigidifications, and are thus widely employed in sensing and imaging applications. ${ }^{11,13,14,36,38,49}$ Below are some examples of their applications in clinical diagnosis.

\section{Cytogenetics studies}

Chromosomes are where genetic materials are held inside cells. Abnormalities in chromosomes, such as changes in chromosome numbers and structures, may lead to birth defects in new-borns, or other health disorders. Clinically, cytogenetic analysis is employed to analyse chromosomal and other related abnormalities, which can provide important diagnostic information and is widely used in oncology, haematology, perinatology, and obstetrics studies. ${ }^{50}$ Classic cytogenetic analyses, such as karyotyping and fluorescence in situ hybridization (FISH), are successful in analyzing chromosome structures, but still have some limitations, especially in the cases of analyzing overlapping chromosomes, and localizing centromere region and gene loci. We recently reported an AIEactive probe, ID-IQ (Fig. 1A), for imaging the chromosome periphery (CP). ${ }^{51}$ To demonstrate the CP specificity of ID-IQ, human embryonic stem (hES2) cells were co-stained with ID-IQ and Hoechst 33342. Hoechst 33342 is a widely used probe for staining DNA, which preferably binds to the adenine-thymine (A-T) regions of DNA. As shown in Fig. 1B, Hoechst 33342 stained the body of the chromosome, while ID-IQ stained the edge of the chromosome. The fluorescences from Hoechst 33342 and ID-IQ complement each other and light up the whole chromosome. In-depth investigations revealed that ID-IQ stains
CP by binding to the proteins in the chromosome periphery. ID-IQ is a general fluorescence probe for CP, and stains other cells as well, such as stem cells (induced pluripotent stem cells, iPSCs), cancer cells (breast cancer cells, MDA-MB-231 and hepatocellular carcinoma cells, HepG2) and normal cells (human embryonic kidney cells, HEK-293T). By labelling the surface of the chromosome, ID-IQ could help us to distinguish overlapping chromosomes and contribute to the segmentation and morphology identification. ID-IQ is compatible with the FISH technique. As shown in Fig. 1C-J, human lymphocyte chromosomes were co-stained by ID-IQ, DAPI and the centromere-specific FISH probe or chromosome $4 \mathrm{q}$ telomere probe. From the ID-IQ channel, the centromere could be clearly identified (Fig. 1G), while it was difficult to differentiate the centromere from the DAPI channel. Compared with the FISH probe, ID-IQ displayed a relatively small spot, demonstrating the higher precision of ID-IQ. Similar conclusions could be drawn when using the chromosome $4 \mathrm{q}$ telomere FISH probe. Such a probe for CP imaging may find practical applications in clinical cytogenetics and quality control in cell therapy manufacturing.

\section{Sperm vitality evaluation}

Sperm vitality reflects the proportion of live spermatozoa and is closely related to human infertility. Human sperm consists of the head, midpiece and tail. The head contains one half of the genetic material, while the tail is thin and long and is responsible for the movement of the sperm. The midpiece contains mitochondria and is responsible for the energy production needed for the movement of sperm. Thus, evaluating the mitochondrial activity of sperm may hint at the mobility of sperm and provide information on the vitality of sperm. ${ }^{52}$ Tang et al. reported a mitochondrion-specific probe and explored its
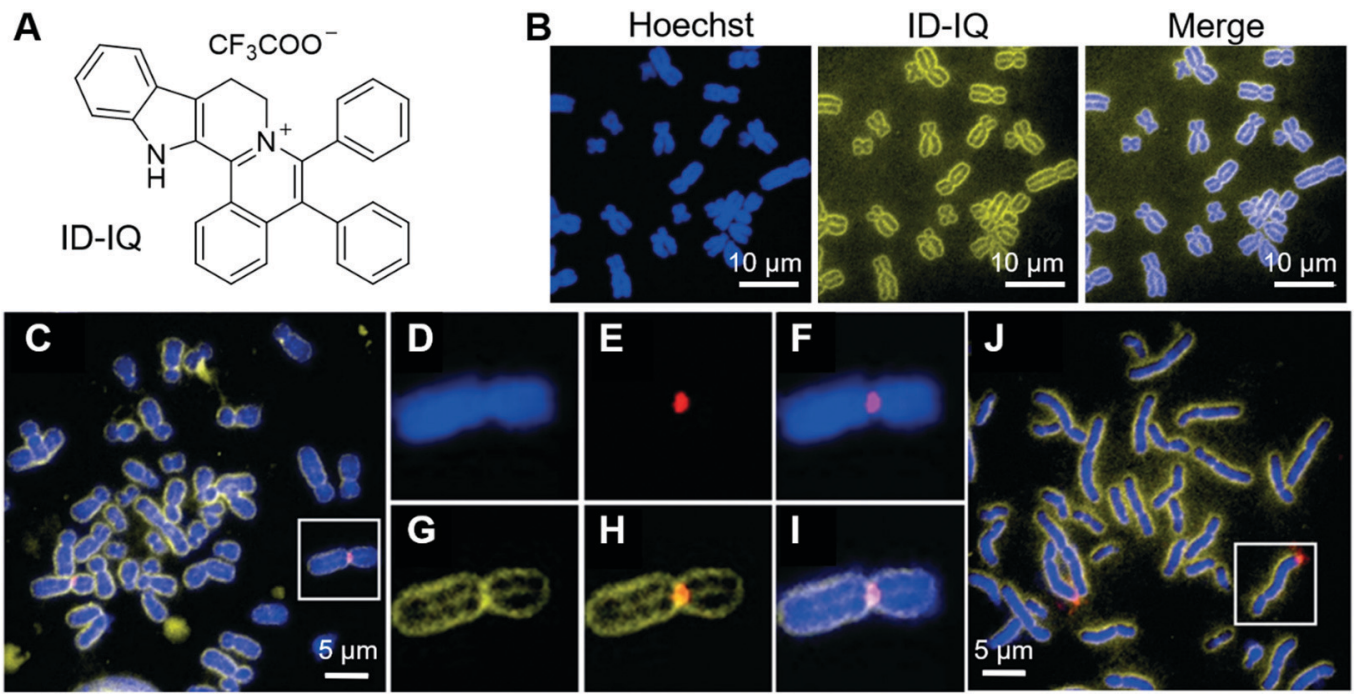

Fig. 1 (A) Molecular structure of ID-IQ. (B) Fluorescence imaging of chromosomes (from hES2 cells) with Hoechst 33342 (blue) and ID-IQ (yellow), in which chromosome bodies were stained with Hoechst 33342 and the chromosome periphery was labelled by ID-IQ. (C-I) Lymphocyte chromosomes co-stained by ID-IQ (yellow), DAPI (blue) and (C) centromere-specific FISH probe (red) or (J) chromosome 4q telomere FISH probe (red). The enlarged views of selected chromosomes (highlighted in white box) in (C) and merged images are given in (D-I). Reproduced with permission from ref. 51. Copyright 2020 Wiley-VCH. 
A
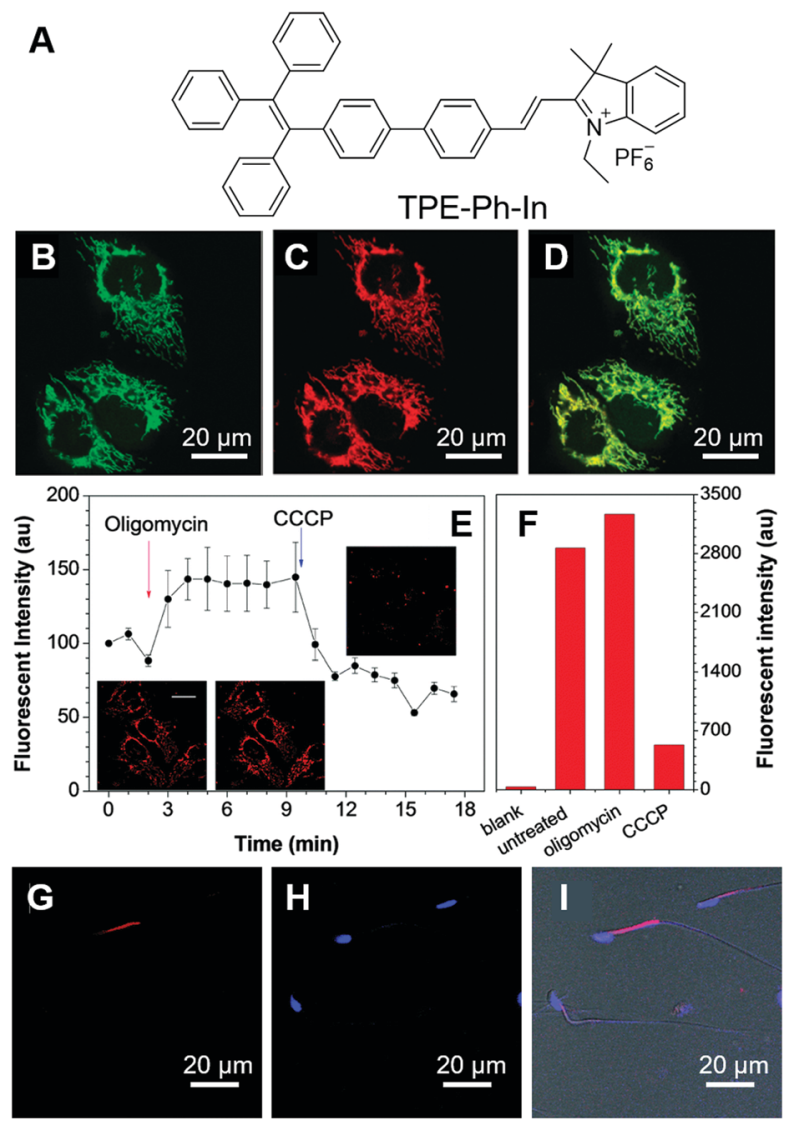

Fig. 2 (A) Molecular structure of TPE-Ph-In. (B-D) Confocal images of HeLa cells co-stained with (B) Mito-GFP, (C) TPE-Ph-In, (D) the merging of panel (B) and panel (C). (E) Changes in the fluorescence intensity of HeLa cells stained with TPE-Ph-In upon treatment with oligomycin and then CCCP. Inset: Snapshots of the cells at different times during the treatment with stimulants. (F) The fluorescence intensity of unstained blank HeLa cells, untreated TPE-Ph-In stained HeLa cells, oligomycin-treated TPE-Ph-In stained HeLa cells and CCCP-treated TPE-Ph-In stained HeLa cells analyzed by flow cytometry. (G-l) Confocal images of mouse sperm cells stained with (G) TPE-Ph-In and (H) Hoechst 33342; (I) the merged picture of $(G),(H)$ and bright-field image. Reproduced with permission from ref. 53. Copyright 2015 The Royal Society of Chemistry.

applications in evaluating sperm vitality. ${ }^{53}$ TPE-Ph-In (Fig. 2A) is a typical AIE-active molecule that fluoresces in the red region. With the positive charge, TPE-Ph-In can easily enter cells and target the mitochondria. To test the specificity of TPE-Ph-In towards mitochondria, HeLa cells were co-stained with TPE-Ph-In and Mito-GFP, which is a commercially available probe specific for mitochondria. As shown in Fig. 2B-D, good colocalizations between these two fluorescent probes were obtained, proving that TPE-Ph-In was indeed mitochondrion specific. Thanks to the AIE attributes of TPE-Ph-In, it could be employed at a relatively high concentration $(5 \mu \mathrm{M})$ and good photostability was obtained. Since the mitochondrial transmembrane potential is the driving force of TPE-Ph-In towards mitochondria, disturbance of the mitochondrial transmembrane potential may affect the accumulation of TPE-Ph-In on mitochondria. To demonstrate this, HeLa cells were firstly treated with oligomycin and carbonyl cyanide 3-chlorophenylhydrazone (CCCP), which could increase and decrease the mitochondrial transmembrane potential, respectively. The fluorescence of TPE-Ph-In became brighter upon oligomycin treatment and dimmer upon the addition of CCCP (Fig. 2E and F). Afterwards, TPE-Ph-In was employed to evaluate sperm vitality. Mouse sperm cells were stained with TPE-Ph-In, and their dynamic motions were tracked and recorded (Fig. 2G-I). The results showed that energetic sperms exhibited bright red emission, while unvital sperms fluoresced faintly or gave no fluorescence. The results suggested that TPE-Ph-In was promising for the evaluation of sperm activity and may be applied to clinical testing.

\section{Detection of circulating tumour cells}

Circulating tumour cells (CTCs) are tumour cells that are detached from a primary tumour and enter the bloodstream. They can travel with the blood and establish new tumours in other sites, which is a well-known process called metastasis. In most cases, metastasis is considered to be the hallmark of malignancies and the main cause of cancer-related death. In this sense, the detection and quantification of CTC is a paramount parameter for the early diagnosis of metastasis. More importantly, CTCs carry critical information on the cancer genotype and deciphering the information may provide guidance for personalized therapy. ${ }^{54}$ Thus, non-invasive methods for CTC detection that exert a minimum effect on cell integrity are highly desirable. Zheng et al. recently reported an AIEgen, TPN (Fig. 3A), for the detection of CTCs. ${ }^{55}$ In contrast to the currently widely employed approaches of cytokeratin (CKs) detection or using FISH to detect the aneuploidy of chromosomes, they selected mitochondria as the target. The mitochondrion is the powerhouse of cells. Tumour cells possess higher metabolic activity than normal cells and can serve as promising anticancer targets. Since leukocytes are the most abundant nucleated cells in the bloodstream, distinguishing between leukocytes and tumour cells is an important criterion for a CTC marker. To demonstrate the feasibility of employing TPN for detecting CTC, leukocytes and seven different tumour cell lines (H1975, A549, SMMC-7721, HepG2, HT-29, MCF-7, and HeLa) were incubated with TPN, respectively. TPN could differentiate tumour cells from leukocytes by the significantly higher emission efficiency from tumour cells (Fig. 3B and C). They also evaluated the influence of TPN-labelling on downstream molecular analysis. Single cells with TPN labelling or immunofluorescence labelling (CKs marker) were isolated and lysed to release intracellular nucleic acids, followed by assessing the single-cell genomic (DNA) or transcriptomics (RNA) information. Compared with traditional immunofluorescence labelling, TPN-labelling retained all the ten genetic loci in all the five single cells, while only 1-2 genetic loci were detected in the 3 out of 5 immunofluorescence-labelled single cells (Fig. 3D). Besides, the labelling of TPN did not interfere with the RNA quality of the single cell, which is in sharp contrast to immunofluorescence labelling. To test the workability of TPN in real samples, whole blood from healthy donors, patients with lung cancer and patients with liver cancer were collected and analysed. CTCs were detected from 53 out of 68 patients with lung cancer, and 11 out of 22 patients with liver cancer, while in healthy donors, only one out of 
A
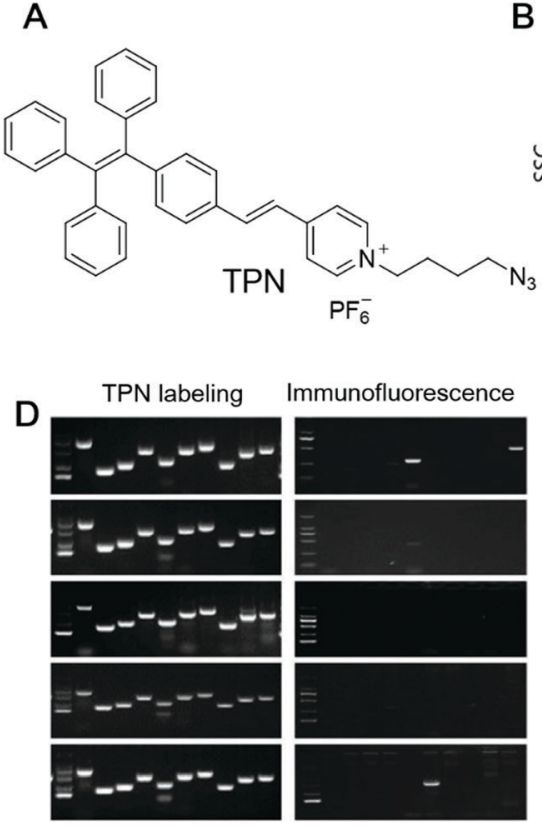

B

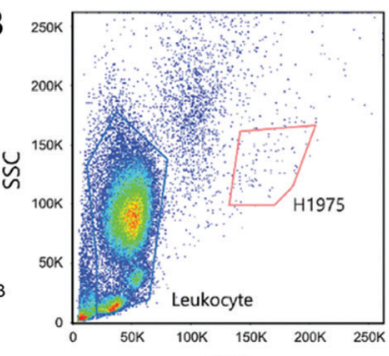

FSC

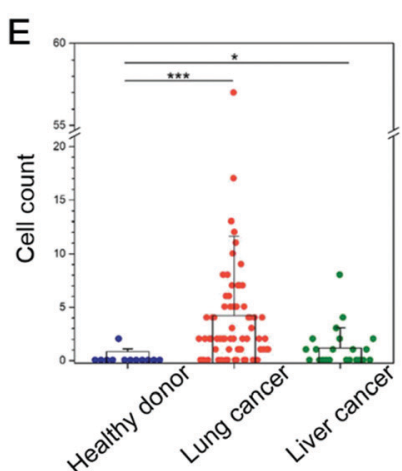

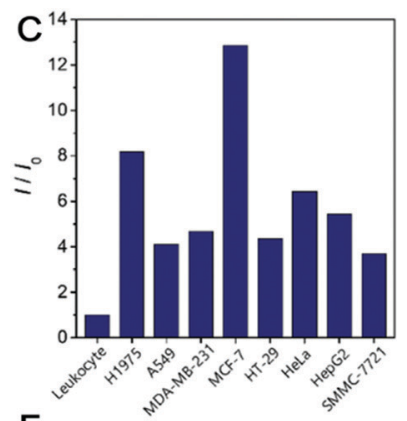

F

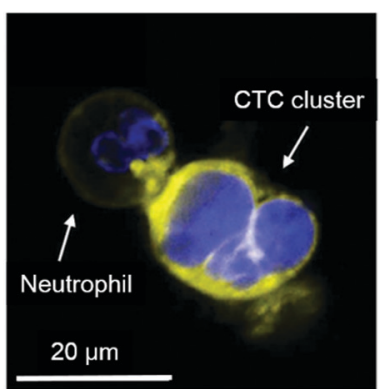

Fig. 3 (A) Molecular structure of TPN. (B) Flow cytometry profile of leukocyte and H1975 cells stained with TPN. (C) Fluorescence intensity of TPN-stained leukocytes and various cancer cell lines measured by flow cytometry. (D) Electropherograms of whole-genome amplification products by MDA from TPN or CKs-labelled single A549 cells assessed by ten genomic loci. (E) Rare tumour cells detected in blood and pleural effusion from cancer patients. Counts of candidate cells in healthy donors, patients with lung cancer, and patients with liver cancer. (F) Fluorescence image of a CTC cluster adhering to a neutrophil in blood. Reproduced with permission from ref. 55. Copyright 2020 Wiley-VCH.

12 patients had two putative cells (Fig. 3E). As shown in Fig. 3F, CTC clusters fluoresced intense yellow light, while neutrophils were almost non-emissive, clearly illustrating that TPN is a very promising candidate for CTC detection.

\section{Intraoperative pathological diagnosis}

Intraoperative pathological diagnosis is crucial for intraoperative decision making, which could distinguish margins from lesions, guide the appropriate resection of tumours, and decide the intraoperative use of a drug. Proper intraoperative pathological diagnosis can improve the patient's outcome, avoid reoperations and lower the cost. ${ }^{56}$ Currently, frozen section consultation is widely employed in intraoperative pathological diagnosis $;{ }^{57}$ the diagnoses have been proven to be accurate, but there are also some limitations, such as the process being timeconsuming and relying on good sampling, careful handling and strong expertise. Dong and Li et al. reported a fast and simple AIE-active stain, ZZ-HPB-NC (Fig. 4A), for the intraoperative pathological diagnosis of hepatocellular carcinoma (HCC), which is a cancer with a high mortality rate and high incidence. ${ }^{58} \mathrm{ZZ}$ HPB-NC can differentiate HCC from normal hepatocytes by selectively lighting up the former. As shown in Fig. 4B, HCC (HepG2, Hep3B and SMMC7721) and normal liver cells (QSG7701 and LO2) were fixed with $4 \%$ paraformaldehyde and co-stained with ZZ-HPB-NC (green fluorescence) and DAPI (blue fluorescence). HCC was rendered with both green and blue fluorescence, while normal liver cells only fluoresced blue light. The results demonstrated that ZZ-HPB-NC was HCC selective and the detailed reason for this selectivity is still under investigation. In complex tumour environments, ZZ-HPB-NC retained this selectivity. Specimens from 14 patients with liver neoplasms, including 11 pairs of HCC foci and paratumour cirrhosis, 3 pairs of focal nodular hyperplasia (FNH) and adjacent were collected and subjected to standard frozen-section preparation protocols, followed by staining with ZZ-HPB-NC. All HCC foci were endowed with the green fluorescence of ZZ-HPB-NC, while other tissues remained unstained. Even more encouragingly, the border between the tumour foci and the adjacent paratumour cirrhosis could be distinguished, with only the tumour foci being stained with green fluorescence and no fluorescence in the paratumour cirrhosis (Fig. 4C), which provided clear guidance for resection. Another advantage of ZZ-HPB-NC staining its highly robust nature. It exhibited good tolerance to sample preparation and even poorly stained or damaged sections could still give informative guidance (Fig. 4D), while intraoperative frozen-section diagnosis was highly dependent on slide preparation. All these results suggest that ZZ-HPB-NC staining is simple, robust and might be applied to intraoperative diagnosis.

\section{Imaging of bone microcracks}

Bones form the major portion of our skeleton and play substantial roles in providing support and structure to our body, protection of the vital organs throughout the body and interaction with muscles for movement. Microcracks on bones are mostly caused by repetitive stress, strain and shear force. Microdamage to the bone can be repaired but unattended bone 

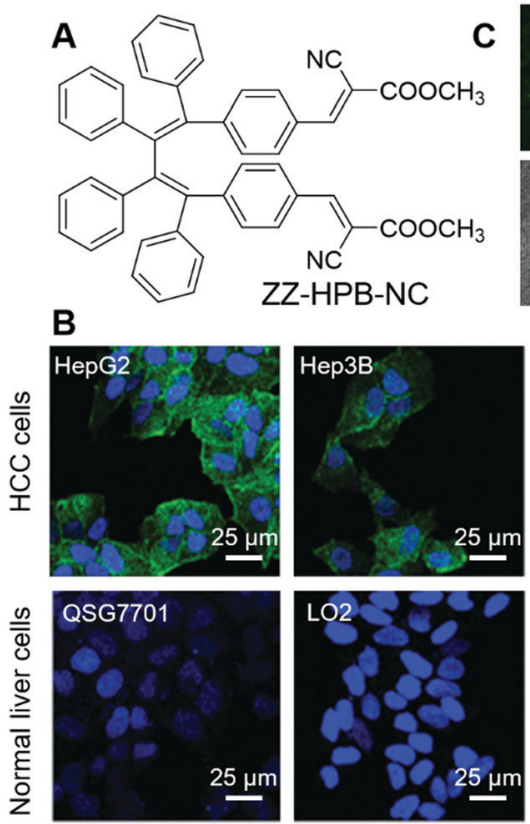

Fig. 4 (A) Molecular structure of ZZ-HPB-NC. (B) Fluorescence images of selected HCC cells and normal cells co-stained with ZZ-HPB-NC (green) and DAPI (blue). (C) Fluorescence image and bright-field image of tumour foci and neighbouring paratumour cirrhosis stained with ZZ-HPB-NC at the same section. (D) Merges of fluorescence and bright-field images of damaged samples stained with ZZ-HPB-NC. Reproduced with permission from ref. 58. Copyright 2020 The Royal Society of Chemistry.

microcracks may lead to a series of fractures and fragmentation. ${ }^{59}$ Tang, Ren and Gao et al. reported an AIEgen for the light-up detection of calcium at high concentrations and employed the probe for imaging microcracks on bones. ${ }^{60}$ SA- $4 \mathrm{CO}_{2} \mathrm{Na}$ (Fig. $5 \mathrm{~A}$ ) is AIE active with $\mathrm{Ca}^{2+}$ specificity. $\mathrm{SA}-4 \mathrm{CO}_{2} \mathrm{Na}$ can dissolve well in an aqueous solution and fluoresce faintly. The iminodiacetate groups on $\mathrm{SA}-4 \mathrm{CO}_{2} \mathrm{Na}$ can chelate with $\mathrm{Ca}^{2+}$ and assist in the formation of highly emissive fibrillar aggregates, resulting in the fluorescence turn-on detection of $\mathrm{Ca}^{2+}$ in the micromolar range (Fig. 5B). The binding of $\mathrm{SA}^{-} 4 \mathrm{CO}_{2} \mathrm{Na}$ towards $\mathrm{Ca}^{2+}$ is highly selective. Other metal ions, including monovalent ions $\left(\mathrm{Li}^{+}, \mathrm{Na}^{+}\right.$, and $\left.\mathrm{K}^{+}\right)$, divalent ions $\left(\mathrm{Zn}^{2+}, \mathrm{Mg}^{2+}\right.$, $\mathrm{Co}^{2+}, \mathrm{Ni}^{2+}$, and $\left.\mathrm{Cu}^{2+}\right)$, and trivalent ions $\left(\mathrm{Fe}^{3+}\right)$, could not turn on the fluorescence of $\mathrm{SA}-4 \mathrm{CO}_{2} \mathrm{Na}$. Although $\mathrm{Mg}^{2+}$ always acted as the interference for $\mathrm{Ca}^{2+}$ detection, $\mathrm{SA}-4 \mathrm{CO}_{2} \mathrm{Na}$ only showed a slight increase in the fluorescence intensity in the presence of $\mathrm{Mg}^{2+}$, suggesting the good selectivity of SA-4CO ${ }_{2} \mathrm{Na}$ towards $\mathrm{Ca}^{2+}$. Apart from sensing $\mathrm{Ca}^{2+}$, SA-4 $4 \mathrm{CO}_{2} \mathrm{Na}$ can also detect $\mathrm{Ca}^{2+}$ in soft tissues. Agarose hydrogel embedded with $\mathrm{CaCl}_{2}$ can also turn on the fluorescence of $\mathrm{SA}-4 \mathrm{CO}_{2} \mathrm{Na}$. Since hydroxyapatite (HA, $\left.\mathrm{Ca}_{10}\left(\mathrm{PO}_{4}\right)_{6}(\mathrm{OH})_{2}\right)$ is the mineral matrix of the bone, and a high concentration of unsaturated $\mathrm{Ca}(\mathrm{II})$ binding sites would be exposed at the bone microcracks, they employed $\mathrm{SA}-4 \mathrm{CO}_{2} \mathrm{Na}$ for the detection of bone microcracks. Previously used fluorescent probes for bone microcracks (such as calcein) suffered from self-quenching and high background noise. Fig. 5C and D show the images of calcein-stained bone microcracks, in which high background emission was present. Although repeated washing
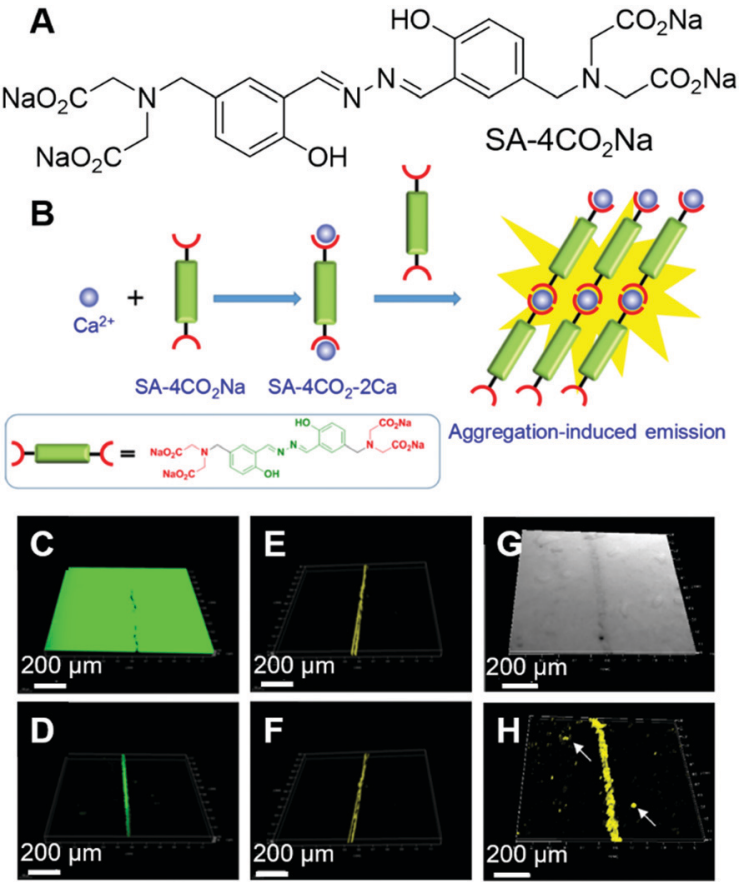

Fig. 5 (A) Molecular structure of $\mathrm{SA}-4 \mathrm{CO}_{2} \mathrm{Na}$. (B) Schematic illustration of the selective detection of $\mathrm{Ca}^{2+}$ by using $\mathrm{SA}-4 \mathrm{CO}_{2} \mathrm{Na}$. (C-F) CLSM images of bovine bone microcracks stained with ( $C$ and $\mathrm{D})$ calcein and ( $\mathrm{E}$ and $\mathrm{F}$ ) $\mathrm{SA}-4 \mathrm{CO}_{2} \mathrm{Na}$. Fluorescence images were collected ( $\mathrm{C}$ and $\left.\mathrm{E}\right)$ without and $(D$ and $F$ ) with washing prior to imaging. $(G)$ Bright-field and $(H)$ fluorescence CLSM images of spot defects and microcracks on the HA-based scaffold stained with $\mathrm{SA}-4 \mathrm{CO}_{2} \mathrm{Na}$. Reproduced with permission from ref. 60. Copyright 2018 American Chemical Society.

could decrease the background, it might also underestimate the seriousness of the bone microcrack. This is because the high local concentration of calcein present in the crack may suffer from selfquenching of fluorescence. In contrast, the $\mathrm{SA}-4 \mathrm{CO}_{2} \mathrm{Na}$ stained bone microcracks in a wash-free manner with low background emission and no self-quenching effect (Fig. $5 \mathrm{E}$ and F). SA- $4 \mathrm{CO}_{2} \mathrm{Na}$ was also applied in detecting microdefects on a HA-based scaffold, from which spot defects and microcracks could be clearly identified (Fig. 5G and H). It was expected that this probe would find broad applications in biomedical studies of $\mathrm{Ca}$ (II) in the micro molar range.

\section{Imaging of atherosclerotic plaques}

Atherosclerosis is the narrowing of the arteries, which could significantly reduce the blood supply to vital organs, such as the heart, brain and intestines, and lead to the blockage of blood and oxygen supply, followed by death or severe damage to the cells. Atherosclerosis is mainly caused by the build-up of fat deposits, called plaques, on the inner surfaces of arteries. Plaques usually consist of cholesterol from low-density lipoproteins (LDL), smooth muscle cells and fibrous tissues, and sometimes calcium. As lipids play crucial roles in the formation of plaques, the evaluation of their amount, location, and distribution in arteries is very necessary. Tang and Zheng et al. recently reported a smart material with AIE attributes, 
namely IND (Fig. 6A), and employed it for the two-photon fluorescence imaging of lipids in mouse atherosclerotic plaques. ${ }^{61}$ IND with two alkyl chains was soluble in sunflower oil with a green fluorescence peaked at $522 \mathrm{~nm}$, while in the water phase it fluoresced red light with an emission maximum of $640 \mathrm{~nm}$. The different fluorescences of IND in water and sunflower oil could be ascribed to their different packing modes. In lipid, IND existed as a monomer and exhibited the fluorescence of a single molecule, while in water, IND was packed as pairwise dimers (Fig. 6B) and thus demonstrated dimer emission. The two alkyl tails of IND were designed to target lipids, following the principle of "like dissolves like". As expected, IND could selectively light up the lipid droplets inside cells with good biocompatibility and in a dosedependent manner. IND also exhibited two-photon absorption with a cross-section of $33.4 \mathrm{GM}$ at $900 \mathrm{~nm}$, making it suitable for the imaging of lipid droplets with two-photon excitation. As shown in Fig. 6C, green spots from lipid droplets could be visualized under two-photon excitation. Inspired by the successful

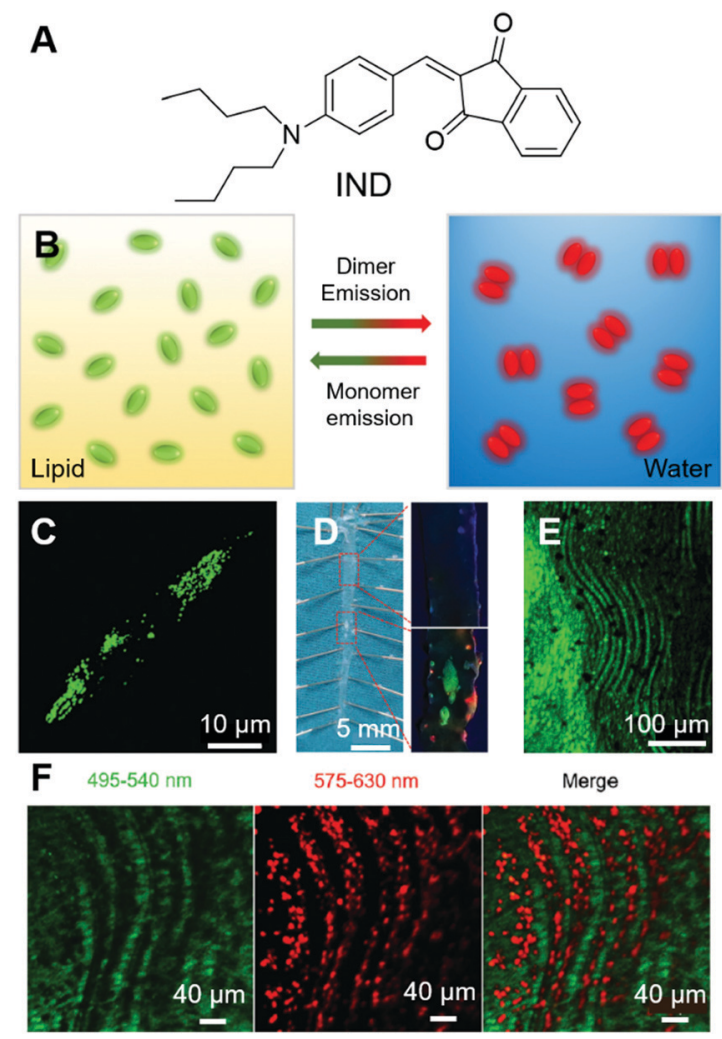

Fig. 6 (A) Molecular structure of IND. (B) Schematic illustration of the transformable fluorescence behaviour of IND in lipid and water. (C) Twophoton image of live foam cells stained by IND after pre-treatment with oxLDL. (D) (Left) En face photograph of the opened aorta taken under daylight. (Right) Fluorescence images of (up) normal artery and (down) atherosclerotic plaques at high magnification taken under $365 \mathrm{~nm}$ UV illumination. (E) Two-photon fluorescence image of lipid accumulation in the aorta of an ApoE gene knockout mouse labelled with IND. (F) Simultaneous dual-colour imaging of the microstructures within the lumen with separated emission ranges of 495-540 nm and 575-630 nm under single two-photon excitation $(900 \mathrm{~nm})$. Reproduced with permission from ref. 61. Copyright 2019 The Royal Society of Chemistry. attempts, they further tested IND by using an animal model with atherosclerosis. The aortic tree of a mouse was separated, unfolded longitudinally and stained with IND. The normal arterial structure was left unstained, while the atherosclerosis region was stained with green fluorescence (Fig. 6D). Taking advantage of the intrinsic advantages of two-photon imaging, such as low background emission and large imaging depth, IND was employed for two-photon imaging of the tissue. Despite the rich collagens in the artery, which had strong autofluorescence, IND lit up the atherosclerotic plaque with high contrast (Fig. 6E). Besides, two-photon imaging with IND enabled a large depth of $\sim 400 \mu \mathrm{m}$. Interestingly, under two-photon excitation, red fluorescence was observed from small aggregates aligned between fatty lines, exhibiting red and green stripes around the plaques (Fig. 6F). The red emission stripe was from IND dissolved in water, which was introduced during the imaging process. This effect demonstrated the high sensitivity of IND and implied its potential applications in revealing biological microenvironments. By employing two-photon excitation, three-dimensional (3D) images of atherosclerotic plaque could be reconstructed, which enabled the direct visualization of the lipid localization, distribution and morphology in the plaque.

\section{Theragnosis}

Theragnosis combines therapy with diagnosis, which enables the simultaneous detection and treatment of the disease. Theragnosis in fluorescent materials is mostly achieved by the combination of fluorescence imaging with PDT. Increasing numbers of AIEgens have been reported to be photosensitive and employed in PDT. ${ }^{62-66}$ Several attributes make AIE PSs excellent candidates for PDT: (a) AIE PSs show increased ROS sensitizing efficiency in the solid or aggregated state, which is in sharp contrast to most traditional PSs with decreased ROS sensitizing efficiency in the aggregated state or at high concentrations. (b) AIE PSs can be employed at high concentrations or as nanoparticles, which endows them with good photostability. Strong long-term light irradiation is generally employed in PDT. Good photostability enables them to continuously sensitize ROS generation and result in better therapeutic effectiveness. (c) Nanoparticles fabricated from AIE PSs can be further decorated with surface functionalities to endow them with good specificity. (d) AIE PSs usually exhibit broad absorption bands, making them excellent candidates for room-light PDT. (e) Most AIE PSs possess low toxicity in the dark and exert minimum toxicity to normal cells. In the following section, we will introduce some representative works employing AIE photosensitizers for theragnosis.

\section{Theragnosis of bacterial infection}

Bacteria are unicellular prokaryotes that exist in enormous numbers and in almost every environment on Earth. Although most bacteria are benign to humans, some infectious bacteria are harmful or even fatal. Antibiotics represent classic treatments for bacterial infection. However, one critical issue of employing antibiotics for bacterial infection is drug resistance. The issue is becoming increasingly serious due to the abuse of antibiotics. 
Antibacterial PDT is a hot research topic, which utilizes the light irradiation of PSs to activate ROS generation. ROS can destroy bacterial structures and inactivate the bacteria. Since the mechanism of ROS inactivating bacteria is irregular and aggressive, it is almost impossible for bacteria to generate resistance.

The development of AIE PSs is a hot research topic. AIEgens bound to bacteria can light up the bacteria and kill them upon light irradiation. Zhu and Lu et al. recently reported an AIEgen with far-red/near-infrared fluorescence for imaging and PDT killing of bacteria. ${ }^{67}$ AIE-TEA (Fig. 7A) fluoresced intensely in the aggregated state with an emission maximum of $678 \mathrm{~nm}$. AIE-TEA could generate ROS upon irradiation with white light. The ROS sensitizing efficiency of AIE-TEA was positively correlated with the degree of aggregation, which changed from $11 \%$ to $83 \%$ by varying the water content of the solvent mixture from $70 \%$ to $95 \%$. AIE-TEA could selectively bind to and light up Gram-positive $(\mathrm{G}(+))$ bacteria over Gram-negative $(\mathrm{G}(-))$ bacteria. Since AIE-TEA can dissolve in water at low concentrations, it enabled the wash-free imaging of bacteria with negligible background emission. Arising from the selectivity of AIE-TEA, it can eliminate $\mathrm{G}(+)$ bacteria more efficiently than killing $\mathrm{G}(-)$ bacteria. $S$. aureus and $E$. coli were incubated with varying concentrations of AIE-TEA and subjected to light/dark treatment. The viable bacteria were quantified by counting colony-forming units (CFU). Upon incubation with $20 \mu \mathrm{M}$ of AIE-TEA, followed by irradiation with while light, almost all the $S$. aureus were killed, while $E$. coli retained around $30 \%$ of CFU ratio at a high AIE-TEA concentration of $40 \mu \mathrm{M}$ with the same duration and power of white-light irradiation (Fig. 7B and C). The antibacterial effect of AIE-TEA was characterized by staining with propidium iodide (PI), a cell membrane-impermeable dye that can selectively enter dead bacteria and mammalian cells through their compromised membrane and endow them with red fluorescence. $S$. aureus incubated with AIE-TEA, followed by treatment with light irradiation glowed intensely, while in the control group, bacteria only exhibited negligible fluorescence. The results proved that AIE-TEA exerted antibacterial PDT through destroying the integrity of the bacterial membrane. The antibacterial effect of AIE-TEA on bacteria was even more obvious when the bacteria were subjected to examination under SEM. The bacterial membranes of $S$. aureus treated with AIE-TEA and light irradiation were shrunken and fused, while the bacterial membranes of $S$. aureus in the control group or $E$. coli with a similar treatment group were almost not affected with distinct edges and smooth surfaces (Fig. 7D-I). The results demonstrated the killing effect of AIE-TEA and presented its promising applications for the treatment of bacterial infections.

From a molecular structure perspective, PSs with donor- $\pi-$ acceptor $(\mathrm{D}-\pi-\mathrm{A})$ structures possess a narrow energy gap $\left(\Delta E_{\mathrm{st}}\right)$ between the excited singlet state $\left(S_{1}\right)$ and excited triplet state $\left(\mathrm{T}_{1}\right)$, which can facilitate the intersystem crossing (ISC) and are favourable for enhancing ROS generation efficiency. Tang and Qin et al. recently reported an AIE-active conjugated polymer for efficient PDT on bacteria. ${ }^{68}$ Benefiting from the strong lightharvesting ability of conjugated polymers, PTB-APFB (Fig. 8A) was designed and synthesized. For comparison, its monomer, MTB-APFB, was also prepared and its antibacterial effect was evaluated. They first measured the ROS sensitizing efficiency of PTB-APFB by employing $2^{\prime}, 7^{\prime}$-dichlorofluorescein (DCFH) as a probe. The ROS sensitizing efficiency of PTB-APFB was 13-fold higher than that of MTB-APFB and 11-fold higher than that of chlorin E6 (Ce 6), which is a commonly employed PS. PTB-APFB could bind to different microorganisms, such as $S$. aureus $(\mathrm{G}(+))$, E. coli $(\mathrm{G}(-))$ and $C$. albicans (fungi) and light them up with red fluorescence (Fig. 8B). PTB-APFB also demonstrated
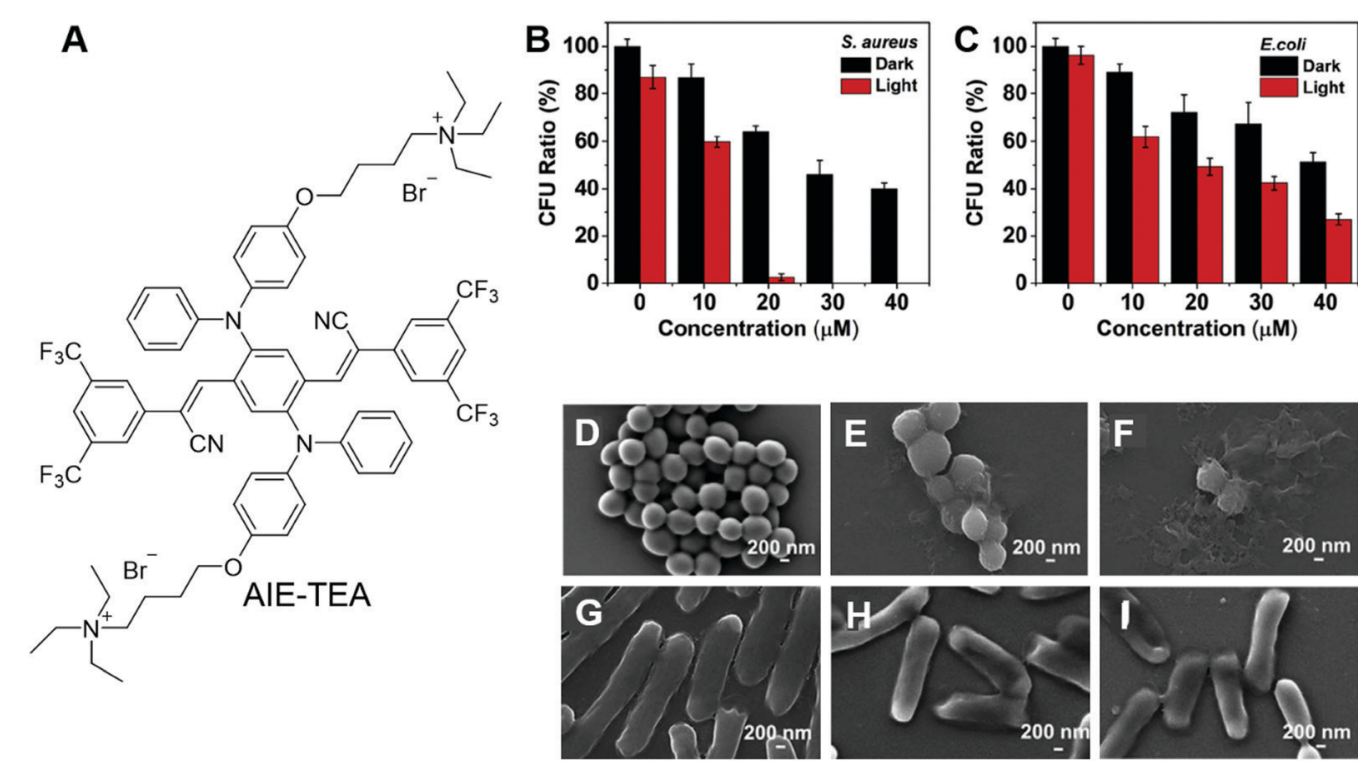

Fig. 7 (A) Molecular structure of AIE-TEA. (B and C) Antimicrobial ability of AIE-TEA against (B) S. aureus and (C) E. coli in the absence and presence of white-light irradiation. The bacteria were incubated with AIE-TEA at varied concentrations for 30 min prior to light irradiation. (D-I) SEM images of (D-F) S. aureus and $(G-I) E$. coli $(D$ and $G)$ without and $(E, F, H$ and $I)$ with incubation with AIE-TEA (E and $H)$ in the dark or ( $F$ and I) under light irradiation for 15 min. Reproduced with permission from ref. 67. Copyright 2019 Elsevier Ltd. 


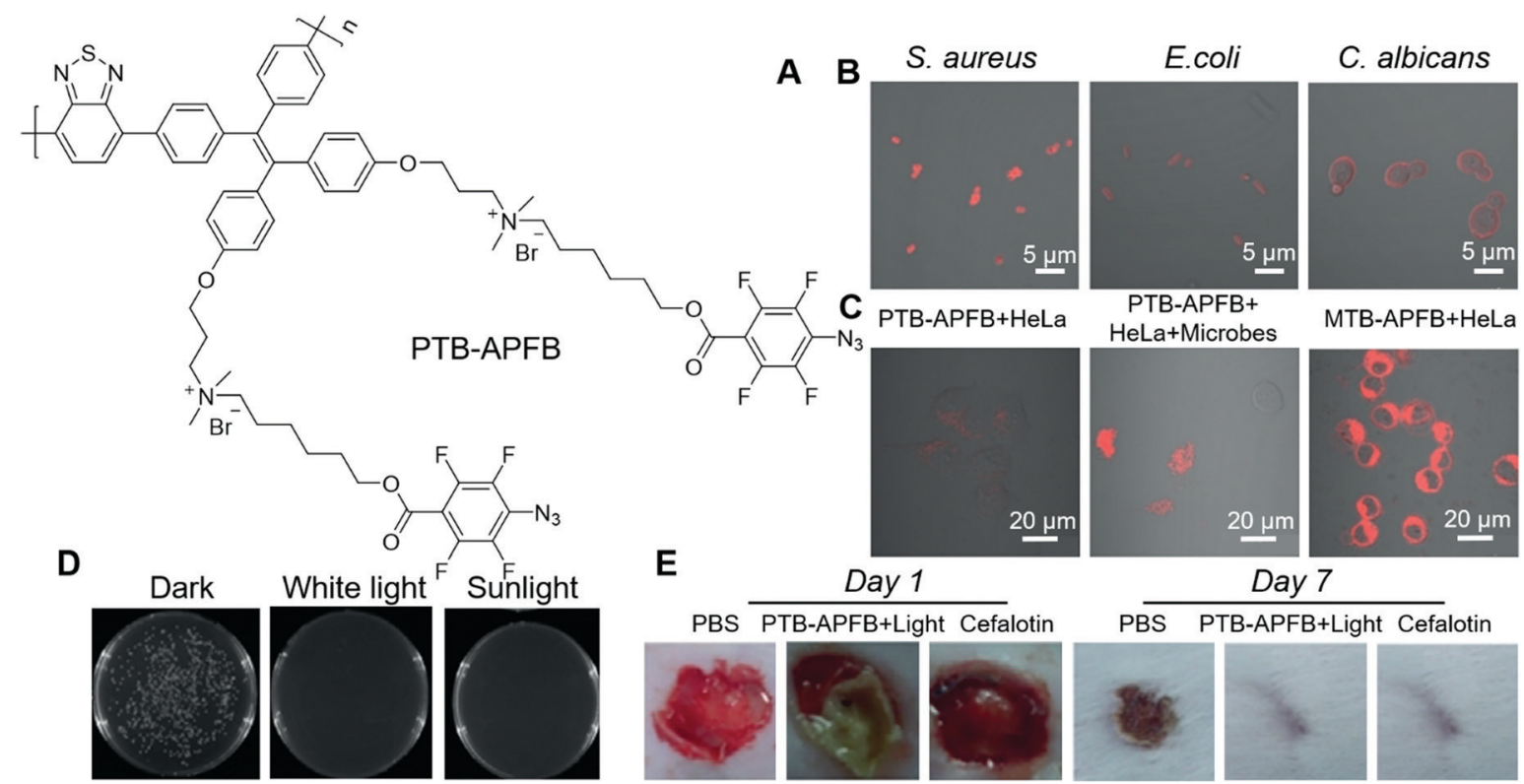

Fig. 8 (A) Molecular structure of PTB-APFB. (B) Merged CLSM images of S. aureus, E. coli, C. albicans incubated with PTB-APFB. (C) CLSM images of HeLa cells and a mixed sample (HeLa cells + microorganisms) incubated with PTB-APFB or MTB-APFB, respectively. (D) Photographs of NB agar plates of S. aureus with PTB-APFB treatment in the dark, and under white light and sunlight. (E) Photographs of the $S$. aureus-infected skin of mice during treatment with the different formulations. Reproduced with permission from ref. 68. Copyright 2020 Wiley-VCH.

excellent biosafety, and the HeLa cell viability remained over $80 \%$ after incubation with $32 \mu \mathrm{M}$ of PTB-APFB for $24 \mathrm{~h}$, while HeLa cells treated with MTB-APFB were less than $10 \%$ viable under the same treatment. Such a distinct difference in toxicity could be due to their variance in interaction with cells. As shown in Fig. 8C, PTB-APFB can selectively bind to microbes over mammalian cells, while MTB-APFB can enter mammalian cells. The antibacterial PDT effect of PTB-APFB was firstly evaluated on $S$. aureus by the plate-count method. Incubating $S$. aureus with PTB-APFB in the dark exerted no effect on its growth, while involving white-light irradiation effectively inhibited its growth (Fig. 8D). Inspired by these successes, they further evaluated the antibacterial effect of PTB-APFB on a mouse model with $S$. aureus infection. The infected mice treated with PTB-APFB-mediated PDT was almost recovered after 7 days, which was 3 days faster than those treated with cefalotin (Fig. 8E). In contrast, infected mice treated with PTBAPFB or white-light irradiation could not fully recover even after 10 days. These results clearly demonstrated the great potential of employing PTB-APFB as an antibacterial agent for preclinical research and clinical applications.

\section{Theragnosis of cancer}

Cancer cells differ from normal cells in many ways. Normal cells could become cancer cells when critical mutations take place and the cells could continuously grow and divide uncontrollably. According to a report from the WHO, cancer is the second leading cause of death globally. Thus, the treatment of cancer has long been a pursuit of scientists. Theragnosis of cancer is becoming increasingly popular. Below are some examples of employing
AIEgens as fluorescence reporters as well as PSs for the theragnosis of cancer cells.

In the PDT of cancer, one very important issue is the penetration depth. The excitation light should pass through the skin and tissue to reach the PSs, while the fluorescence from theragnostic PSs should also penetrate through the skin and tissue to be detected. PDT employing two-photon excitation with NIR light for excitation are advantageous. Qian and Zhang, et al. recently reported an AIEgen for two-photon PDT. ${ }^{69}$ TPE-red (Fig. 9A) was fabricated into nanoparticles, TPE-red-PSMA, by ultrasonicating the THF mixture of TEP-red and poly(styrene-co-maleic anhydride) (PSMA). TPE-red-PSMA was obtained with an average particle size of $\sim 64.1 \mathrm{~nm}$ and a low dispersity, and could sensitize ROS generation upon light irradiation. Besides, TPE-red-PSMA demonstrated deep-red two-photon emission upon irradiation with a $1040 \mathrm{~nm}$ fs laser. The two-photon absorption cross-section of TPE-red was determined to be $27.72 \mathrm{GM}$ at $1040 \mathrm{~nm}$. They further conducted two-photon fluorescence imaging of HeLa cells. As shown in Fig. 9B and C, TPE-red-PSMA could enter the cells and endow HeLa cell with red fluorescence. The nanoparticles could also sensitize ROS generation upon two-photon excitation, and exert a PDT effect on cancer cells. To demonstrate the phototoxicity of TPE-red-PSMA upon two-photon excitation, HeLa cells were incubated with TPE-red-PSMA nanoparticles and fluorescein isothiocyanate (FITC)-tagged Annexin V (Annexin V-FITC). Annexin V-FITC can selectively bind to phosphatidylserine (PS), which exists in the inner membrane of healthy cells and is exposed to the outer membrane during apoptosis or necrosis; thus, Annexin V-FITC could track apoptotic or necrotic cells by lighting them up. As shown in Fig. 9D-F, cells with both TPE-redPSMA staining and two-photon irradiation with a $1040 \mathrm{~nm}$ fs laser 

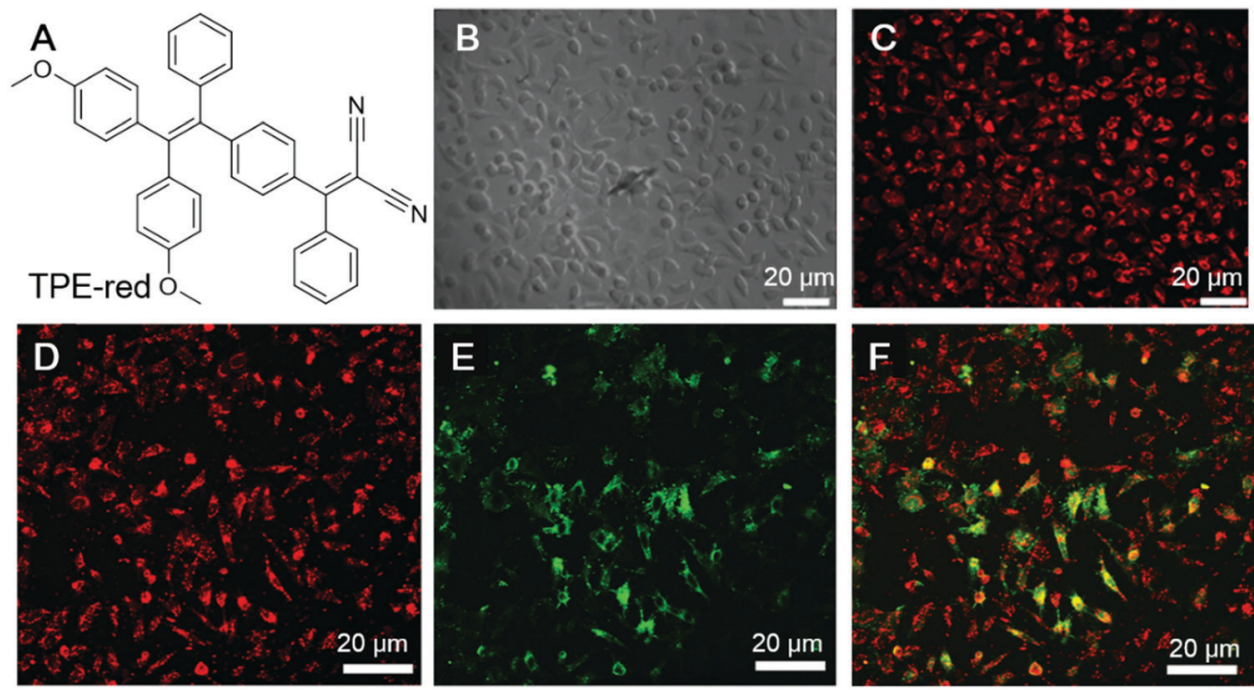

Fig. 9 (A) The molecular structure of TPE-red. (B and C) Bright-field and two-photon fluorescence images of HeLa cells treated with TPE-red-PSMA nanoparticles: (B) bright-field image of HeLa cells, and (C) the corresponding two-photon fluorescence image under $1040 \mathrm{~nm}$ fs laser excitation. (D-F) Measurement of the rate of two-photon excitation induced apoptosis of HeLa cells. Cells were treated with both TPE-red-PSMA nanoparticles and Annexin V-FITC. (D) Two-photon fluorescence from TPE-red-PSMA nanoparticles in the red channel. (E) Two-photon fluorescence from Annexin V-FITC in the green channel. (F) Overlap of the red and green channels. Reproduced with permission from ref. 69 . Copyright 2017 The Royal Society of Chemistry.

for $120 \mathrm{~s}$ displayed strong green fluorescence, indicating the good PDT effect of TPE-red-PSMA. The cell death ratio was evaluated to be about $90 \%$. In the control group treated with TPE-red-PSMA alone or Annexin V-FITC alone, no fluorescence could be detected from the green channel. This work clearly demonstrated the feasibility of employing two-photon irradiation for PDT, which overcame the penetration depth issue.

Bryce and Tang et al. recently reported the applications of AIE-active nanoparticles for cancer imaging and therapy. ${ }^{70}$ Two PSs with AIE attributes were designed and synthesized (Fig. 10A). By molecular engineering, PS1 and PS2 were designed with a D-A structure, which could lower the $\Delta E_{\mathrm{st}}$, facilitate the ISC and lead to high ROS sensitizing efficiency. The $\Delta E_{\text {st }}$ of PS1 and PS2 were $0.229 \mathrm{eV}$ and $0.204 \mathrm{eV}$, respectively. The smaller $\Delta E_{\mathrm{st}}$ of PS2 was ascribed to its additional triphenylamine functionality, which led to the smaller spatial overlap of the frontier orbitals. Indocyanine green (ICG) was used as the indicator for the evaluation of the ${ }^{1} \mathrm{O}_{2}$ sensitizing efficiency. The ${ }^{1} \mathrm{O}_{2}$ quantum efficiency of PS1 and PS2 were determined to be $38 \%$ and $43 \%$, respectively. Their aggregates demonstrated even higher ${ }^{1} \mathrm{O}_{2}$ sensitizing efficiencies, which was $67 \%$ and $76 \%$ for PS1 nanoparticles and PS2 nanoparticles, respectively. The PDT effects of these PSs were evaluated by MTT colorimetric assay. Both PS1 and PS2 showed good biocompatibility, with almost no dark toxicity on HeLa cells (Fig. 10B). In the presence of white light irradiation, both PS1 and PS2 exhibited a dose-dependent PDT effect. A similar PDT effect was observed on other cell lines as well, such as MDA-MB-231 cells and A 549 cells. More interestingly, both PS1 nanoparticles and PS2 nanoparticles demonstrated fast cell staining and long-term retention inside cells. As shown in Fig. 10C, the red fluorescence of PS1 could be observed from HeLa cells after incubation for as short as 5 seconds. After 1 min, the fluorescence was very strong. This enabled washing-based imaging. Once inside cells, PS1 and PS2 could be retained for more than 15 days, owing to the good biocompatibility and cell retention of these probes, which enabled long-term cell imaging and were beneficial for therapeutic applications. They further evaluated the in vivo PDT effect by employing U14 tumourbearing mice as targets. After injection with PS1 nanoparticles but without light treatment, the tumour site retained $42 \%$ emission intensity, while for the experimental group with both PS1 and light irradiation, the fluorescence was rather faint due to the elimination of the tumour (Fig. 10D). During the 14 days of experiment, the tumour size in the control group grew by 10-13 times, while that in the experimental group showed a significant reduction (Fig. 10E). The results unambiguously demonstrated its excellent PDT effect.

\section{Strategies for improving PDT outcome}

In the above examples, the research focus was mostly on molecular engineering to design and synthesize PSs with high ROS sensitizing efficiency and long emission wavelength. There are also some other strategies for improving the PDT outcome. In the next section, we select some examples to illustrate these strategies.

One intrinsic advantage of PDT is the precision of treatment, which could avoid interferences to normal cells by manipulating the site of irradiation. However, in one-photon PDT, strong light scattering is involved, which lowers the spatial precision on the $z$-axis. In contrast, in two-photon PDT, two strong lasers with ultra-high photon densities are employed to selectively excite the PSs in the focal point, and achieve better control of PS activation in 3D. Tang and Zheng et al. recently reported stereotactic PDT by employing two-photon PSs. ${ }^{71}$ DPASP (Fig. 11A) was AIE-active with a red emission band peaked at $646 \mathrm{~nm}$ and a large Stokes 
A<smiles>N#CC(C#N)=C(c1ccccc1)c1ccc(-c2ccc(N(c3ccccc3)c3ccccc3)cc2)cc1</smiles><smiles>N#CC(C#N)=C(c1ccc(-c2ccc(N(c3ccccc3)c3ccccc3)cc2)cc1)c1ccc(N(c2ccccc2)c2ccccc2)cc1</smiles>

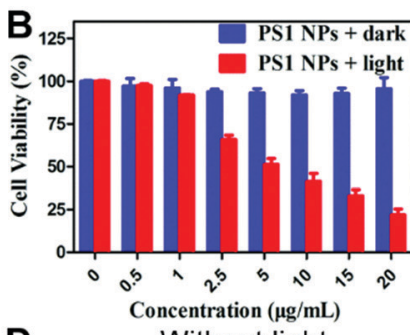

Without light

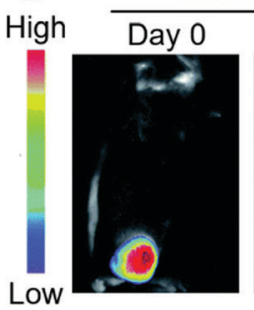

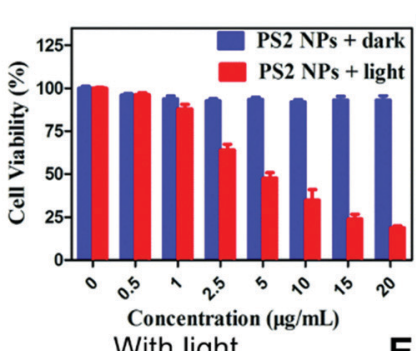

5 sec
$1 \mathrm{~min}$

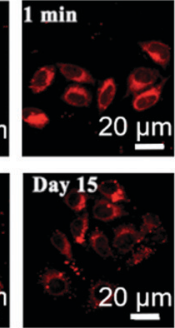

$\mathrm{E}$
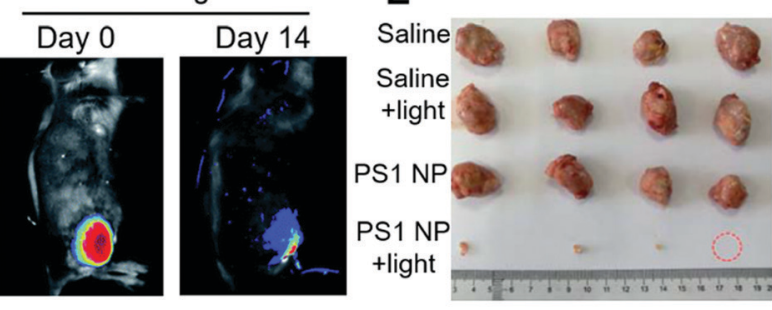

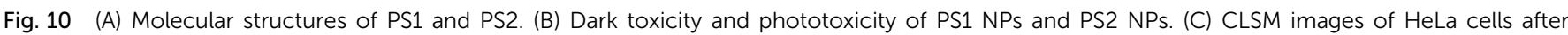

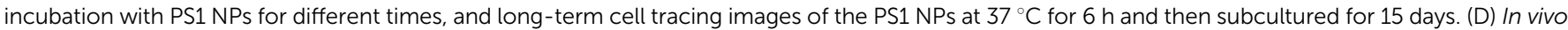

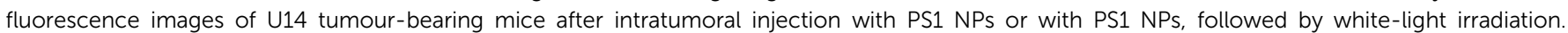
(E) Harvested tumours from the various treated groups. Reproduced with permission from ref. 70 . Copyright 2020 The Royal Society of Chemistry.

shift of $189 \mathrm{~nm}$, which was beneficial for alleviating self-absorption and enhancing the signal-to-noise ratio. With the positive charge in molecular structure, DPASP could facilely enter cells and target the mitochondria of cells. To demonstrate this, human non-small
A

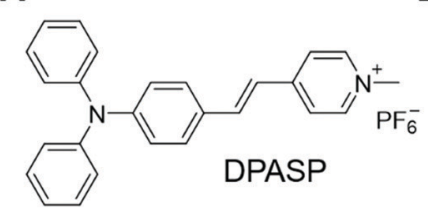

B

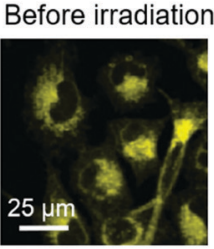

C

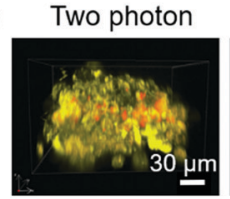

D

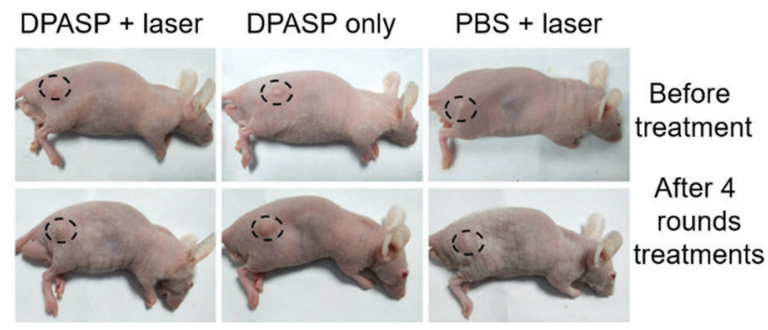

E

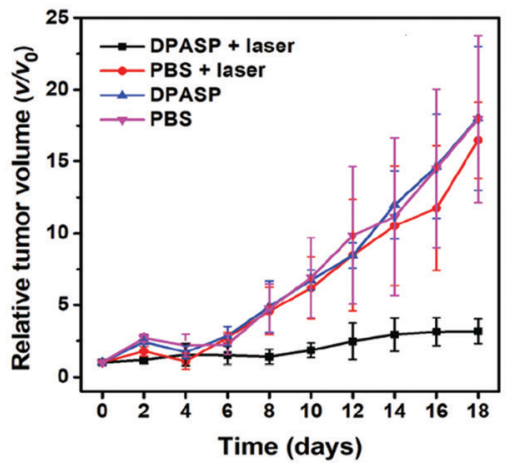

Fig. 11 (A) Molecular structure of DPASP. (B) Two-photon images of A549 cells treated with DPASP and PI before and after $960 \mathrm{~nm}$ irradiation. (C) 3D reconstructed two-photon and one-photon images of the A549 MCTS after stereotactic irradiation at the depth of $60 \mu \mathrm{m}$ with $960 \mathrm{~nm}$ laser. (D) Photographs of tumour-bearing mice before treatment and after four rounds of treatments. (E) Relative changes in the tumour volumes of the A549 tumour-bearing nude mice of the different groups after various treatments. Reproduced with permission from ref. 71 . Copyright 2019 Wiley-VCH. 
cell lung cancer cells (A549 cells) were co-stained with DPASP and MitoTracker Deep Red (MT), and subjected to imaging with confocal microscopy. The overlap coefficiency and Pearson's correlation coefficient were calculated to be 0.90 and 0.77 , respectively, which substantially proved the mitochondrion targeting of DPASP. DPASP demonstrated a large two-photon absorption cross-section of $235 \mathrm{GM}$ at $960 \mathrm{~nm}$, which enabled its practical applications to sensitize ROS generation by excitation with $960 \mathrm{~nm}$ lasers. The two-photon therapeutic effect of DPASP was evaluated by co-staining A549 cells with DPASP and PI, followed by irradiation with a $960 \mathrm{~nm}$ laser. As shown in Fig. 11B, after irradiation with the $960 \mathrm{~nm}$ laser, red fluorescence of PI was observed in the cell nucleus, demonstrating the therapeutic effect of DPASP. To evaluate the 3D precision of DPASP, multicellular tumour spheroids (MCTs) were stained with DPASP and PI, and subjected to one-photon PDT and twophoton PDT at the depth of $60 \mu \mathrm{m}$. By reconstructing the images on the $z$-axis, clear 3D images were obtained. As shown in Fig. 11C, in two-photon PDT, dead cells were mostly aligned in the same plane, while in one-photon PDT, dead cells could be discerned at all the depths. The results distinctly demonstrated the higher 3D precision of two-photon PDT over one-photon PDT. They further assessed the in vivo two-photon PDT on A549 bearing mice. With two-photon PDT, the relative tumour volume was also unchanged, while in all the control groups the relative tumour volume was substantially increased (Fig. 11D and E), which demonstrated the high effectiveness of DPASP-mediated two-photon PDT.

PSs with limited tumour selectivity always require avoiding normal exposure to white light, as they will activate the PSs on the skin and exert toxicity to patients. The issue could be relieved by increasing the specificity of PSs. Li and Wang et al. reported another approach for improving the precision in PDT of incorporating PSs in liposomes. ${ }^{72}$ In their approach, PSs, such as BP and MC4 (Fig. 12A), were embedded in liposomes (BP@liposomes and MC4@liposomes), which could inhibit the photosensitivity of the PS. Upon reaching tumour cells, these liposomes were degraded and the PSs could reaggregate and recover their photosensitivity (Fig. 12B). In this way, the phototoxicity of PDT on normal tissue would be reduced. Taking BP@liposomes as an example, they first evaluated the assembly and disassembly process in cells. MCF-7 cells were employed as models and incubated with BP@liposomes. As the incubation time was prolonged from $4 \mathrm{~h}$ to $30 \mathrm{~h}$, the green fluorescence increased accordingly, which was ascribed to the degradation of BP@liposomes and reaggregation of these BP molecules (Fig. 12C). In contrast, as the incubation time was extended to $30 \mathrm{~h}, \mathrm{MCF}-7$ incubated with nano-BP fluoresced faintly. Besides, the extremely large two-photon absorption cross-section of BP@liposomes $\left(2.4 \times 10^{5} \mathrm{GM}\right)$ was beneficial for two-photon PDT. 9,10-Anthracenediyl-bis(methylene)dimalonic acid (ABDA), DCFH-DA and phosphorescence spectra were employed to verify

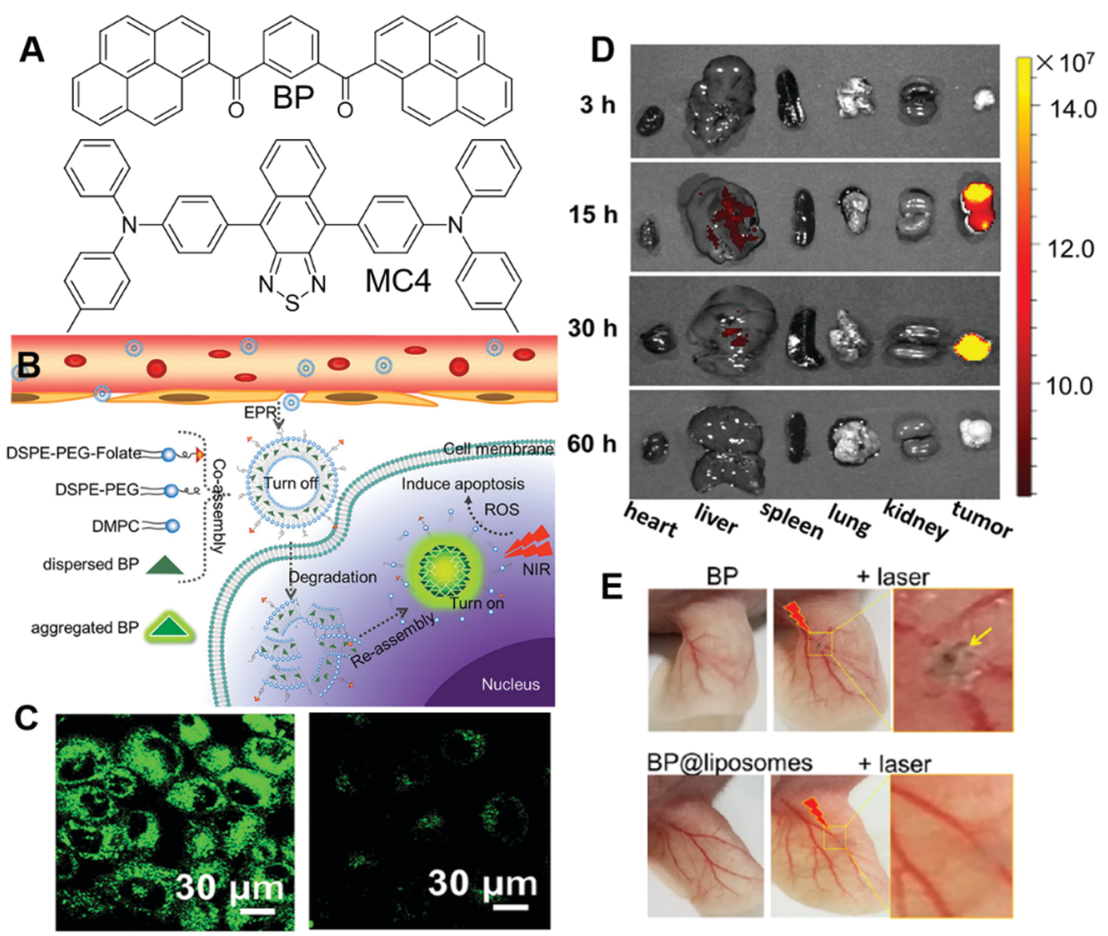

Fig. 12 (A) Molecular structures of BP and MC4. (B) Illustration of co-assembled AIE-PS@liposomes composed of AlE-PS and lipids for antitumour treatment in PDT. (C) Two-photon CLSM images of cells co-cultured with (left) BP@liposomes doped with folate lipid and (right) nano-BP. (D) Fluorescence images of the major organs (heart, liver, spleen, lungs, and kidneys) resected from mice post-injection of MC4@lipsomes-folate at 3, 15, 30, and 60 h. (E) Blood vessels in the ears being injected with nano-BP, BP@aliposomes, followed by two-photon laser irradiation treatment (middle and right images) or without irradiation treatment (left image). The ears in mice whose entire bodies were exposed under sunlight for $2 \mathrm{~h}$ after injection with BPaliposomes. Reproduced with permission from ref. 72. Copyright 2019 American Chemical Society. 
the ROS sensitizing ability of BP@liposomes, which proved its high ROS sensitizing efficiency. They also demonstrated that BP could sensitize ROS production more efficiently as aggregates or nanoparticles than in dispersed states, which was consistent with previous reports. The two-photon PDT effect of BP@liposomes was evaluated on MCF-7 cells by using PI as an indicator. In cellular experiments, folate-conjugated lipid and PEG-lipid were added to AIE@liposomes to prepare the AIE@liposome-folate for improving their specificity to tumours and increasing their retention time in tumours, respectively. Upon irradiation with the twophoton laser, the red fluorescence of PI emerged in the cells gradually, which substantially proved the PDT effect of BP@liposomes-folate. Encouraged by these positive results, they further evaluated the PDT effect in vivo. Since BP emits in the green region and was not suitable for deep-tissue penetration, MC4 with deep red emission was fabricated into MC4@liposomes-folate and employed for in vivo imaging experiments. As shown in Fig. 12D, MC4@liposomes-folate selectively accumulated in the tumour over other organs during $15 \mathrm{~h}$ to $30 \mathrm{~h}$ post-injection, while other organs showed faint or no fluorescence. These AIE@liposomes particles also demonstrated excellent PDT effects as expected with significantly smaller tumour sizes than the control groups after 20 days of treatment. Furthermore, the phototoxicity of BP@liposomes was evaluated by irradiating mice ears with a laser. Mice receiving nano-BP presented severe burns with shrunken skin, while mice receiving BP@liposomes were not affected by laser irradiation under the same conditions (Fig. 12E). The phototoxicity of commercial PS Ce6 was also evaluated for comparison, which possessed much higher phototoxicity than the BP@liposomes, with blood vessels in the ear turning brown and damaged under the same conditions. Such strategies were promising for liberating patients from the dark room during and after PDT.

The design of PSs could be very challenging, which calls for mechanistic insights. It would be ideal if we could control the ROS-sensitizing efficiency through engineering approaches rather than molecular design. Tang and Ding, et al., reported a novel strategy for boosting the PDT efficiency by incorporating corannulene into AIE nanodots. ${ }^{73}$ In their work, TPP-TPA (Fig. 13A) was employed as the PS, which absorbed at $440 \mathrm{~nm}$ and fluoresced in the red region with an intense emission peak at $680 \mathrm{~nm}$. TPP-TPA was then fabricated into nanoparticles through nanoprecipitation. Cor-PEG with bowl-shaped corannulene and DSPE-PEG (Fig. 13A) were employed as encapsulant matrixes to prepare Cor-AIE dots and DSPE-AIE dots, respectively. The particle sizes of the as-prepared Cor-AIE dots and DSPE-AIE dots were determined to be $46.9 \mathrm{~nm}$ and $49.1 \mathrm{~nm}$, respectively. Despite their similarity in particle size, their properties were distinctly different. Cor-AIE dots exhibited stronger emission with a quantum yield of $26.8 \%$ and a longer fluorescence lifetime (4.34 ns) as compared to DSPE-AIE dots. Besides, Cor-AIE dots also possessed higher ROS sensitizing ability, with ROS sensitizing efficiency being 4.4 times higher than that of DSPE-AIE dots. The reason for the elevated ROS sensitizing efficiency of TPP-TPA in Cor-AIE dots was carefully investigated by ${ }^{1} \mathrm{H}$ NMR and density functional theory (DFT)

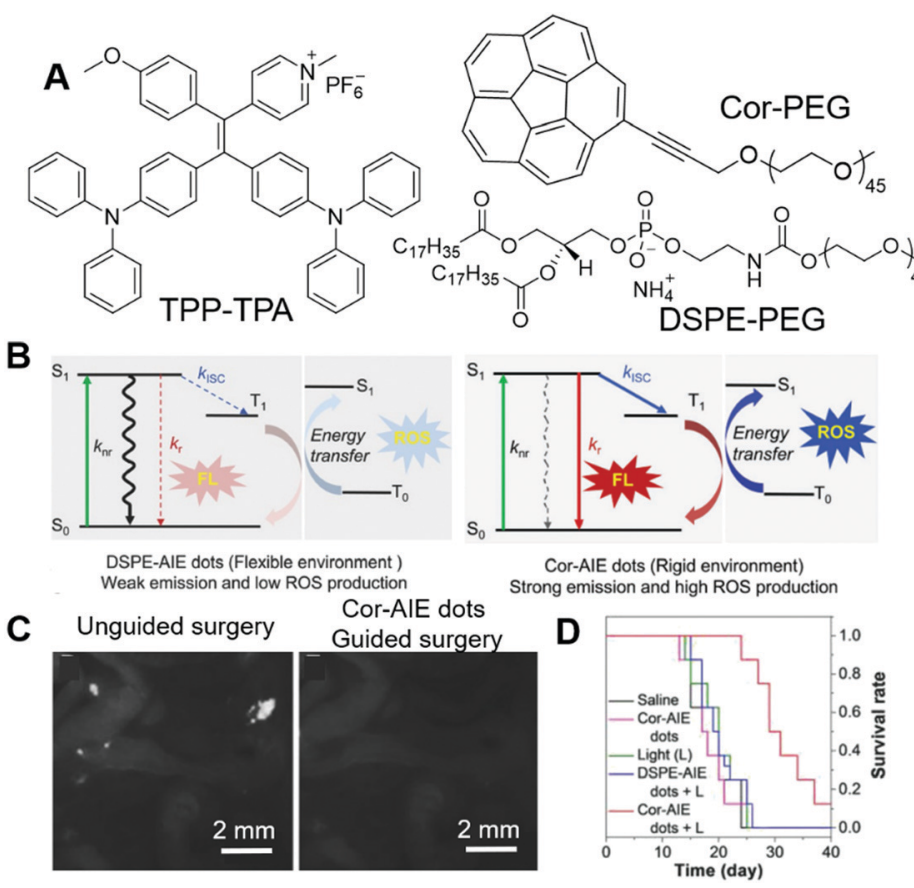

Fig. 13 (A) Molecular structures of TPP-TPA, Cor-PEG and DSPE-PEG. (B) Jablonski diagram showing the nonradiative, radiative, and intersystem crossing (ISC) processes for AIEgens in flexible (DSPE-AIE dots) and rigid (Cor-AIE dots) matrixes. $\mathrm{S}_{0}$ : the ground state, $\mathrm{S}_{1}$ : the lowest excited singlet state, $\mathrm{T}_{1}$ : the lowest excited triplet state. $k_{\mathrm{nr}}, k_{\mathrm{r}}$, and $k_{\mathrm{ISC}}$ are the rate constants of the nonradiative relaxation, the radiative decay and the ISC process, respectively. (C) Representative fluorescence images after unguided surgery, and after reoperation with the aid of Cor-AIE dots image-guidance. (D) Survival rates of mice after different treatments. Reproduced with permission from ref. 73. Copyright $2018 \mathrm{Wiley-VCH.}$ 
calculations, from which a mechanistic picture was revealed. Interacting with corannulene could lower the $\Delta E_{\mathrm{st}}$, and increase the respective spin-orbital coupling constants between $\mathrm{S}_{1}$ and $\mathrm{T}_{1}$, which would favour the ISC and result in higher ROS generating efficiency (Fig. 13B). From the perspective of molecular structure, corannulene with a large dipole moment was electronegative at the bottom of its bowl structure, which would attract positively charged TPP-TPA through dipole-dipole and electrostatic interactions. Such interactions confined TPP-TPA, restricted its molecular motions and favoured ISC, which promoted ROS sensitization. Benefiting from the near-infrared fluorescence of TPP-TPA, it could be employed in imagingguided surgery. Cor-AIE dots were then intravenously injected into mice bearing murine 4T1 cancer cells, which could express luciferase and emit bioluminescence when administrated with its substrate (D-luciferin). The bioluminescence signal from luciferase overlapped perfectly with the NIR fluorescence signal of Cor-AIE dots, demonstrating the tumour targeting of Cor-AIE dots. After $24 \mathrm{~h}$, surgery was performed by opening the abdomens of the mice. Guided by Cor-AIE dots, scattering intraperitoneal tumour nodules could be totally and specifically lit up, which ensured the differentiation and complete removal of small tumour nodules with sizes smaller than $1 \mathrm{~mm}$ (Fig. 13C). Highcontrast imaging-guided surgery avoided the recurrence of tumours. In the cases where surgery was not performable, PDT with Cor-AIE dots could be performed to suppress tumour growth. As shown in Fig. 13D, with Cor-AIE dots-mediated PDT, the survival rates of the mice were significantly prolonged, while DSPE-AIE did not have an observable inhibitory effect on tumour growth. This study opened a new avenue for enhancing the fluorescence and ROS sensitizing efficiency of AIE dots.

Besides the approaches to increasing PDT efficacy, research efforts have also been devoted to combining PDT with other therapies, such as photoacoustic imaging, and photothermal therapy, to achieve multimodal theragnosis. ${ }^{63}$ The synergetic effects of different modes could greatly enhance the therapeutic effect, and avoid resistance issues. Tang and Wang et al. prepared an AIE-active photosensitizer combined with 2 dimensional (2D) black phosphorus and applied it in the multimodal theragnosis of tumours. ${ }^{74}$ Recently, they prepared a one-for-all AIEgen for PDT, photothermal therapy, and photoacoustic imaging, which could precisely reveal tumour locations and completely eliminate tumours. ${ }^{75}$ Furthermore, Ding et al. reported the highly efficient induction of immunogenic cancer death through imposing focused mitochondrial oxidative stress with AIEgen, demonstrating the feasibility of AIEgen for immunity therapy. ${ }^{76}$ Readers interested in these advances may refer to recent reviews. ${ }^{62,63,77}$

\section{Mechanism studies and personalized medicine}

One of the most intriguing attributes of fluorescent materials is their ability to present biological processes and provide mechanistic insights into these processes. AIEgens with good photostability, non-invasive attributes, and simple and easy operation were thus employed for mechanism studies, and providing guidance for personalized medicine.

\section{Bactericidal mechanism}

Scientists are always keen about antibacterial materials. The underlying mechanism of how these antibacterial agents combat bacteria varies with the type of these antibacterial materials. Mechanistic insight into these processes may provide guidance on the use of these materials and may also contribute to the further development of new antibacterial materials. Tang, Wang and Ren, et al. reported the applications of AIEgens for investigating the bactericidal mechanism of antimicrobial peptides. ${ }^{78}$ They decorated antimicrobial peptide HHC-36 (KRWWKWWRR) with AIE-active HBT to prepare AMP-2HBT (Fig. 14A). AMP-2HBT inherited the AIE attribute of HBT. The fluorescence of AMP-2HBT could be turned on upon binding with bacteria. Due to the low background emission of AMP-2HBT, there was no need for involving washing procedures to remove unbound AMP-2HBT molecules. Besides, AMP-2HBT also demonstrated similar antimicrobial activity to

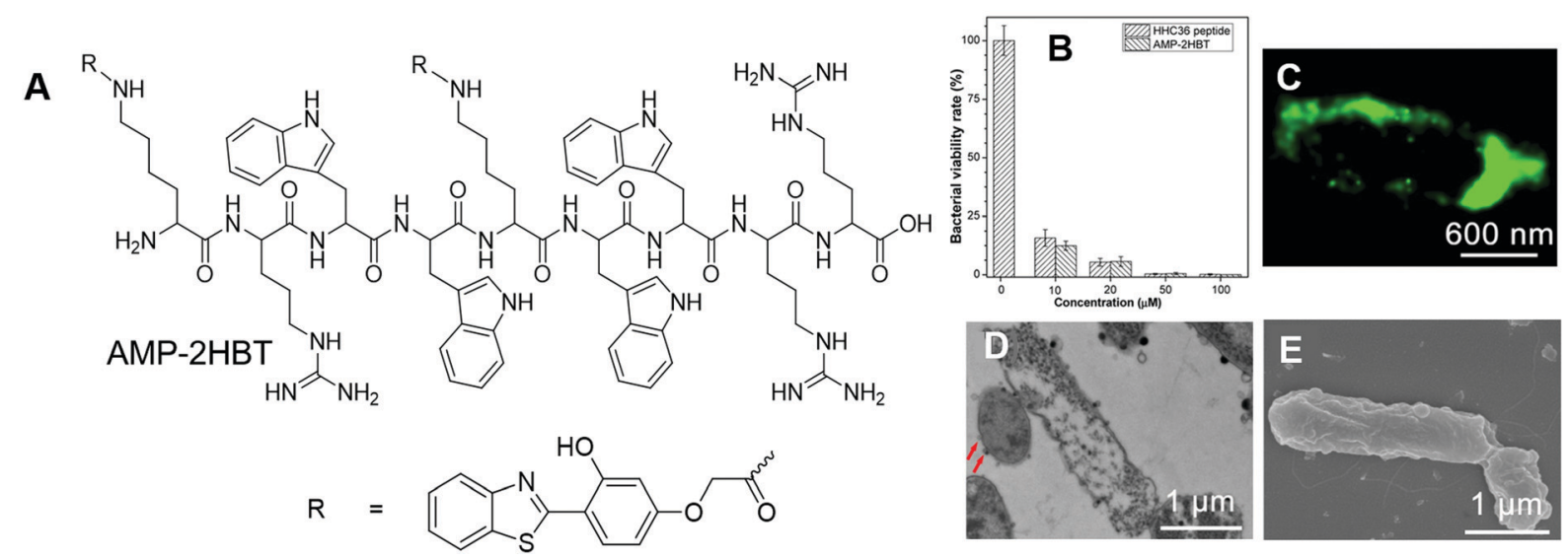

Fig. 14 (A) Molecular structure of AMP-2HBT. (B) Antimicrobial ability of HHC36 peptide and AMP-2HBT against E. coli at different concentrations. (C) Super-resolution fluorescence image, (D) TEM image and (E) SEM image of E. coli after treatment with AMP-2HBT. Reproduced with permission from ref. 78. Copyright 2018 American Chemical Society. 
the HHC36 peptide (Fig. 14B), which proved that decorating HHC36 with HBT did not interfere with the antibacterial activity of the HHC 36 peptide. Upon incubation with AMP$2 \mathrm{HBT}$, the fluorescence of bacteria increased with the incubation time, while no fluorescence was observed from bacteria incubated with HBT with the carboxyl functionality. The results proved that the binding of AMP-2HBT towards bacteria originated from the HHC36 peptide. To precisely locate the spatial distribution of AMP-2HBT, super-resolution fluorescence imaging was conducted with stochastic optical reconstruction microscopy (STORM). As shown in Fig. 14C, strong but discontinuous fluorescence was observed on the bacterial membrane, which suggested that the probe tended to reside on the bacterial membrane with high density. Besides, for bacteria treated with AMP-2HBT, hollow structures were observed in the TEM image (Fig. 14D) and a rough membrane structure was identified in the SEM image (Fig. 14E). The results pointed to the process that HHC36 accumulated on the cell membrane, and disrupted the membrane structure, which induced the leakage of bacterial DNA and proteins.

Most antibacterial materials work better on $\mathrm{G}(+)$ bacteria than on $\mathrm{G}(-)$ bacteria. That is because the $\mathrm{G}(-)$ bacterial cell envelope contains an additional outer membrane, which could serve as an additional protective barrier to prevent the entry of toxic compounds. Thus, it is more challenging to design antibacterial materials against $\mathrm{G}(-)$ bacteria. Tang, et al. recently reported their investigations on modulating the antibacterial effect by adjusting molecular charges on PSs. ${ }^{79}$ TBP-1 and TBP-2 with the same chromophore but different charges were designed and synthesized (Fig. 15A). The photosensitizing abilities of these two AIEgens were characterized by using ABDA as an indicator as well as electron spin resonance (ESR). The decomposition rates of ABDA were calculated to be $18.2 \mathrm{nmol} \mathrm{min} \mathrm{m}^{-1}$ and $15.3 \mathrm{nmol} \mathrm{min}^{-1}$ for TBP-1 and TBP-2, respectively. Their PDT effects were evaluated on both $\mathrm{G}(+)$ and $\mathrm{G}(-)$ bacteria. S. epidermidis $(\mathrm{G}(+))$, S. aureus $(\mathrm{G}(+))$, E. coli $(\mathrm{G}(-))$ and $E$. coli TOP10 (G(-)) were chosen as representatives. Among them, E. coli TOP10 is an ampicillin-resistant strain. Both TBP-1 and TBP-2 exerted dose-dependent dark toxicity on $\mathrm{G}(+)$ bacteria and could effectively eliminate $\mathrm{G}(+)$ bacteria upon light irradiation. However, TBP-1 could not kill the two $G(-)$ bacteria strains both in the dark and with light irradiation (Fig. 15B, left). In contrast, TBP-2 was not toxic to $G(-)$ bacteria in the dark but could eliminate $\mathrm{G}(-)$ effectively in the presence of light (Fig. 15B, right). SEM was then employed to investigate the reason for the different performances of TBP-1 and TBP-2. As shown in Fig. 15C, S. epidermidis incubated with TBP-1 and TBP-2 showed a deformed and collapsed morphology. The structure of $E$. coli stained with TBP-1 remained unchanged, while that incubated with TBP-2 displayed a deformed morphology. From the CLSM results, both TBP-1 and TBP-2 could stain $\mathrm{G}(+)$ bacteria, while only TBP-2 could stain $\mathrm{G}(-)$ bacteria and endow it with strong fluorescence. The results revealed the following mechanistic pictures: $\mathrm{G}(+)$ bacteria cytoplasm membranes were covered by a thick and porous cell wall of peptidoglycan and anionic teichoic acids, which could attract positively charged molecules. $\mathrm{G}(-)$ bacteria with an additional outer membrane could prevent

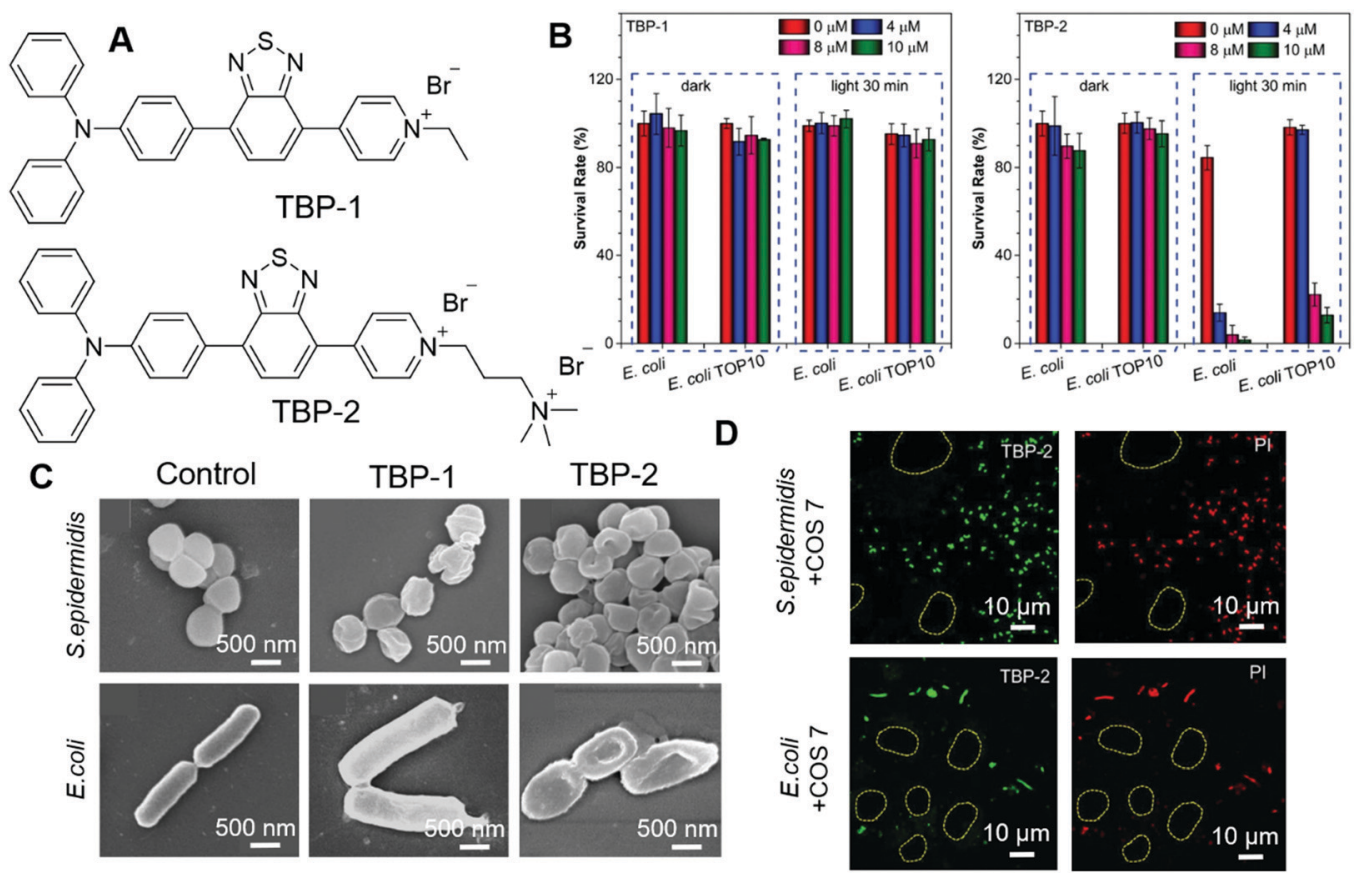

Fig. 15 (A) Molecular structures of TBP-1 and TBP-2. (B) Antibacterial activity of TBP-1 and TBP-2 towards Gram (-) bacteria (E. coli and E. coli TOP10). The bacteria were incubated with TBP-1 or TPB-2 at various concentrations for 10 min, followed by remaining in dark or white-light irradiation. (C) SEM images of S. epidermidis and E. coli incubated with TBP-1 or TBP-2 for 10 min, then irradiated by white light. (D) Confocal images of the mixed COS-7 cells with S. epidermidis or E. coli after incubation with TBP-2 for 10 min followed by white-light irradiation. The mixed cells and bacteria were then stained by $200 \times 10^{-9} \mathrm{M} \mathrm{PI}$ and captured by CLSM to check the selective phototoxicity. Reproduced with permission from ref. 79 . Copyright $2020 \mathrm{Wiley}-\mathrm{VCH}$ 
cationic AIE PSs from disrupting their cell membranes in the dark. TBP-2 with one more positive charge interacted more strongly than TBP-1, and could replace the divalent cations $\left(\mathrm{Ca}^{2+}\right.$ and $\left.\mathrm{Mg}^{2+}\right)$, as revealed by the $\mathrm{Mg}^{2+}$ competition experiment. Furthermore, TBP-2 could selectively stain bacteria over mammalian cells. As shown in Fig. 15D, mixtures of COS 7 and E. coli or S. epidermidis were incubated with TBP-2, and PI was employed to examine the PDT effect. Only bacteria were stained with green fluorescence of TBP-2, while mammalian cells (circles) remained unstained. Treatment with light only killed the bacteria rather than mammalian cells. This work revealed the importance of positive charges on PSs and could guide the development of more efficient PSs.

\section{Guidance on personalized medicine}

In the treatment of bacterial infections, the sensible selection of suitable antibiotics is of critical importance. The right antibiotics should be the ones that the infected bacteria are susceptible to. Improper prescription of antibiotics may delay the treatment and may exacerbate the issue of antibiotic-resistance. By taking advantage of the high sensitivity and the fluorescence turn-on attribute of AIEgens, we developed a strategy for the fast evaluation of bacterial susceptibility and high-throughput screening of antibiotics. ${ }^{80}$ The molecular structure of AIEgen employed is shown in Fig. 16A. The AIEgen was water-soluble at low concentrations but formed micelles at concentrations above the critical micelle concentration (CMC) $(20 \mu \mathrm{M})$. With the two positive charges, the AIEgen could bind to both $\mathrm{G}(+)$ and $\mathrm{G}(-)$ bacteria, and endow them with deep yellow fluorescence. As the concentration of AIEgen was below CMC, no washing procedure was needed and the bacteria could be visualized with high contrast. The working principle of the strategy was as follows: a certain number of bacteria were cultured in the presence of varied species and concentrations of antibiotics for $4 \mathrm{~h}$, and AIEgen was added at the end of culturing. In the presence of effective antibiotics, bacterial growth would be inhibited and few bacteria would be present, resulting in the weak fluorescence of the solutions; in the presence of ineffective antibiotics, bacteria grew freely and large numbers of bacteria would exist in the culture media, leading to the strong fluorescence of the solutions (Fig. 16B). In this way, the effectiveness of the antibiotics could be evaluated by judging from the fluorescence intensity, and the critical parameters of the antibiotics, such as minimum inhibitory concentration (MIC) and half-maximal inhibitory concentration ( $\left.\mathrm{IC}_{50}\right)$, could be determined (Fig. 16C). Taking advantage of the same strategies, the bacteria susceptibility could also be determined, with susceptible bacteria giving weak fluorescence and resistant bacteria showing strong fluorescence after culturing (Fig. 16D). The new method may contribute to the development of new antibiotics and lay the foundation for personalized diagnosis and prescription of bacterial infection.

\section{Tissue regeneration visualization}

Tissue regeneration describes the renewal and restoration process, through which a damaged or lost body could be rebuilt. Although some human tissues, such as skin, fingertips,

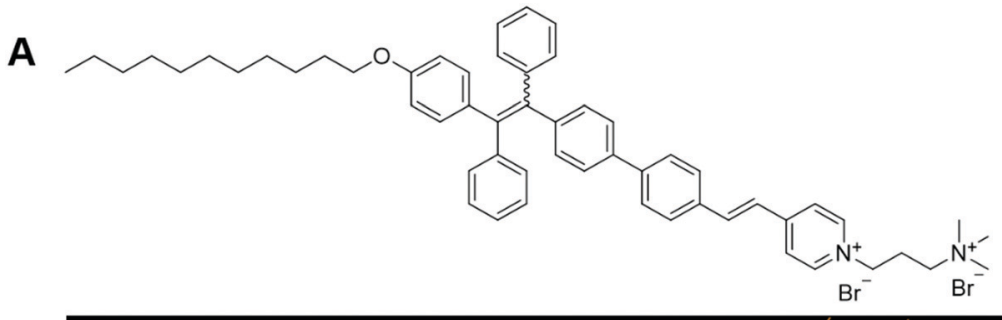

B
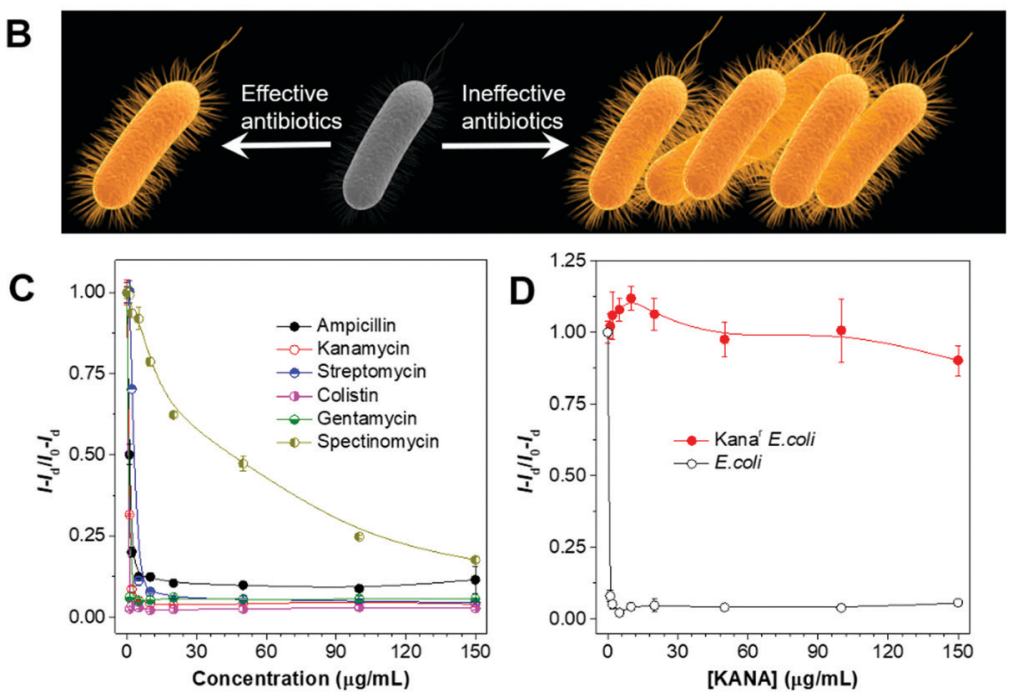

Fig. 16 (A) Molecular structure of the AlEgen employed. (B) Schematic illustration of the process for screening antibiotics and the evaluation of bacterial susceptibility. (C) Evaluation of the effectiveness of antibiotics with AIEgen. (D) Evaluation of the bacterial susceptibility with AIEgen. Reproduced with permission from ref. 80. Copyright 2015 Wiley-VCH. 

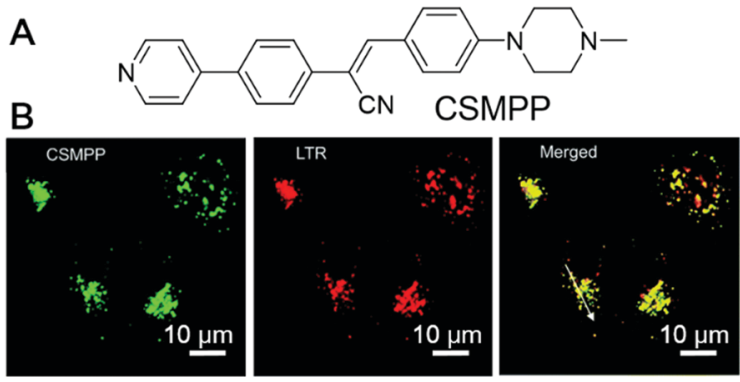

C $\mathrm{pH}_{3}$

$\mathrm{pH} 4.5$

$\mathrm{pH} 6.5$
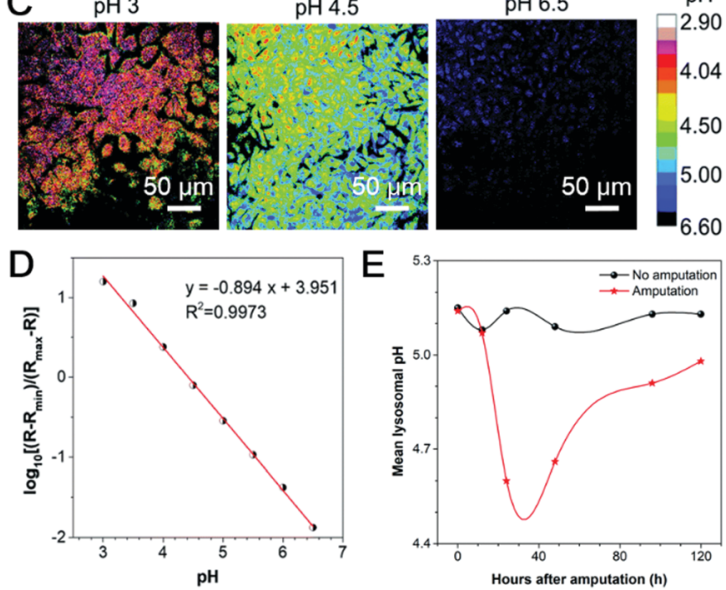

Fig. 17 (A) Molecular structure of CSMPP. (B) Confocal images of HeLa cells stained with Lyso-Tracker Red (LTR) for $10 \mathrm{~min}$ and then co-stained with CSMPP for $10 \mathrm{~min}$. (C) The $\mathrm{pH}$ calibration of the CSMPP probe in zebrafish cells captured using CLSM. (D) The mean ratio of $E_{\mathrm{m} \text {-red }} / E_{\mathrm{m} \text {-green }}$ as a function of $\mathrm{pH}$. (E) The mean lysosomal $\mathrm{pH}$ changes of the medaka larva's caudal fin during regeneration. Reproduced with permission from ref. 82. Copyright 2020 The Royal Society of Chemistry.

endometrium and liver can be regenerated by themselves, the regeneration ability is weak as compared with some vertebrates, such as salamanders, zebrafish, and Xenopus. ${ }^{81}$ Regenerative medicine represents a relatively young field of multidisciplinary research aimed at promoting the repair or regeneration of impaired body organs and tissues. In these research efforts, a critical issue is to evaluate the tissue rebuilding effect. Tang et al. recently reported the utilization of an AIEgen for imaging the tissue regeneration process. ${ }^{82}$ In their approach, lysosomes were selected as the target, due to their important roles in autophagy, a critical process in tissue regeneration. To track the lysosome and quantify lysosomal $\mathrm{pH}, \mathrm{CSMPP}$ was designed and synthesized (Fig. 17A). The piperazine group endowed the probe with lysosome specificity, while the large charge transfer during pyridine protonation enabled ratiometric quantification of lysosome pH. The lysosome specificity of CSMPP was revealed by staining the human retinal pigment epithelial cells (ARPE-19). Lyso-Tracker Red (LTR) was also employed to costain the ARPE-19 cells. The fluorescence of CSMPP and LTR overlapped well with a Pearson correlation coefficient of 0.92 (Fig. 17B). CSMPP demonstrated good photostability as well as excellent biocompatibility, which enabled its application in tracking the tissue regeneration process with minimum interference to the targets. With the pyridine group, CSMPP was able to ratiometrically quantify the $\mathrm{pH}$ changes in lysosomes. At low $\mathrm{pH}$, the pyridine group will be protonated, and become strongly electron-withdrawing. This resulted in the formation of a $\mathrm{D}-\pi-\mathrm{A}$ structure and endowed CMSPP with a reddish fluorescence. In a neutral or alkaline environment, the pyridine group was deprotonated, and the fluorescence of CSMPP was shifted to the blue region. By taking advantage of the fluorescence changes in CSMPP, the varied pH inside cells could be mapped (Fig. 17C). The $\log \left[\left(R-R_{\min }\right) /\left(R_{\max }-R\right)\right]$ and $\mathrm{pH}$ showed a good linear relationship in the $\mathrm{pH}$ range of 3.0-6.5 (Fig. 17D), which was used to calibrate the lysosomal $\mathrm{pH}$ of the caudal fin. Tracking the lysosome $\mathrm{pH}$ during caudal fin regeneration was performed on medaka larva. Medaka larvae were fed with $5 \mu \mathrm{M}$ CSMPP $4 \mathrm{~h}$ prior to the amputation of the caudal fin. CSLM images of medaka larvae before amputation and at 12, 24, 48, 96 and $120 \mathrm{~h}$ post amputation were collected and analysed. The mean $\mathrm{pH}$ of the caudal fin decreased gradually after amputation, and the lowest $\mathrm{pH}$ appeared 24-48 $\mathrm{h}$ after amputation, corresponding to the blastema formation stage (Fig. 17F). At $24 \mathrm{~h}$ after amputation, the pH dropped from 5.1 to 4.6. During this period, autophagy was active to remove damaged tissues and cellular debris. The lysosome $\mathrm{pH}$ increased after $48 \mathrm{~h}$ and returned to normal after $120 \mathrm{~h}$. This work represented a simple and quantitative method for dynamically tracking tissue regeneration.

\section{Bone marrow stromal cell tracking}

Stem cells are undifferentiated cells with the potential to form many different cell types in the body, which could perform specialized functions. By sensing varied chemical signals, they could move into damaged tissue and start the differentiation process, resulting in the healing of damaged tissues. ${ }^{83}$ Mechanistic insight into the way that stem cells promote tissue regeneration is highly desirable, which could direct the development of stem cellbased therapies. Liu and Liao, et al. reported the application of organic nanoparticles fabricated from AIEgens for tracking bone marrow stromal cells (BMSC). ${ }^{84}$ They designed and synthesized an AIEgen, TPEEP (Fig. 18A), with high brightness and low phototoxicity. TPEEP was fabricated into nanoparticles through the nanoprecipitation method. The THF solution of TPEEP and DSPE-PEG $_{3000}$ was rapidly injected into MilliQ water, which resulted in the swift change in the solvent solvating power and led to the aggregation of TPEEP. The hydrophobic DSPE segments with hydrophilic PEG chains extended to the aqueous phase (Fig. 18A). TPEEP NP with an average diameter of $\sim 45 \mathrm{~nm}$ was obtained, as determined by TEM analysis, which demonstrated deep red fluorescence with a broad fluorescence band that peaked at $690 \mathrm{~nm}$ and a high fluorescence quantum yield of $35 \pm 1 \%$. The as-prepared TPEEP NP demonstrated excellent stability in varied environments, such as in the presence of varied proteins, in acidic or alkaline solutions, which was desirable for in vitro and in vivo tracking applications. For tracking applications, cell penetration protein, Tat, was conjugated to the surface of the NPs to enhance the internalization of nanoparticles (TPEEP-Tat NP). After incubation with BMSC, red fluorescence could still be observed after 9 days, indicating the excellent tracking ability of TPEEP-Tat NP. BMSCs labelled with TPEEP-Tat NP were then transfused into a rat 


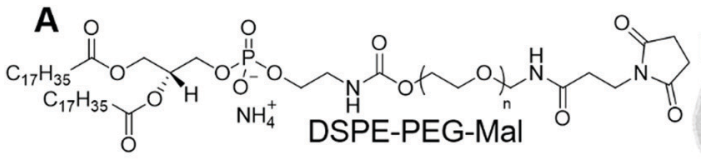<smiles>[B]C1CC1C</smiles>

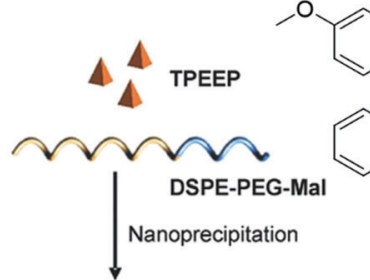<smiles>C=CC(=C)C(=CC)c1ccc(OC)cc1</smiles>
TPEEP
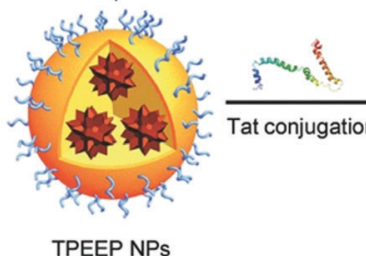

TPEEP NPS
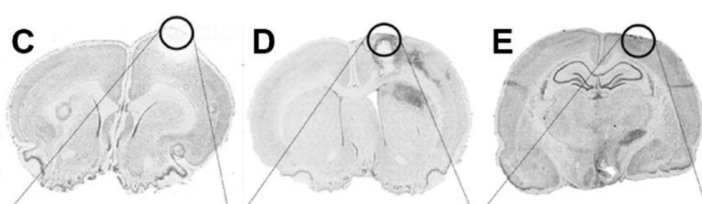

$\mathbf{G}$
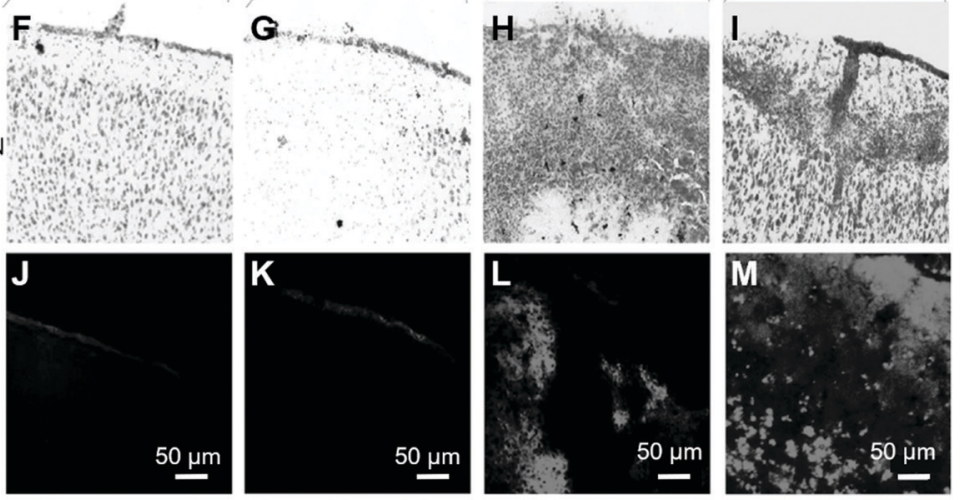

Fig. 18 (A) Molecular structures of TPEEP and DSPE-PEG-Mal and schematic illustration of TPEEP NP preparation. (B-E) Microscopic images of thionin-stained brain slices collected from rats (B) without PTI, (C-E) with PTI and BMSC transfusion, (C) 3 days after BMSC transfusion, (D) 5 days after BMSC transfusion, (E) 7 days after BMSC transfusion. Black circles indicate the lesion sites. (F-I) high magnification images of the circled regions. (J-M) Fluorescence images of brain slices collected from rats (J) without PTI, (K-M) with PTI and BMSC transfusion, (K) 3 days after BMSC transfusion, (L) 5 days after BMSC transfusion, (M) 7 days after BMSC transfusion. Reproduced with permission from ref. 84. Copyright $2016 \mathrm{Wiley-VCH}$.

photothrombotic ischemia (PTI) mode to evaluate their tracking ability in vivo. Ischemic stroke was induced in rats by the PTI technique in a targeted cortical arteriole. Rats with different treatments were perfused with 0.1 M PBS and sacrificed to harvest the brain for histological assessment. In the stroke region of rat brain, less cells presented than the intact region in healthy rats. The brain slice in Fig. 18B and F demonstrated healthy brain slices without PTI. On the third day post-BMSC transfusion, no BMSC could be observed in the ischemic region (Fig. 18C and G). Starting from day 5, BMSCs migrated to and gathered around the ischemic region (Fig. 18D and $\mathrm{H}$ ). On day 7 , the ischemic region was fulfilled with BMSCs, indicating the successful migration and homing of BMSCs in the ischemic region following PTI stroke induction. Bright emission from stem cells could be observed after 5 days of BMSCs transplantation (Fig. 18L and M), while no fluorescence could be discerned in the healthy group (Fig. 18J). Thus, by tracking the presence of red emission from stem cells, the stem cell-therapy process could be visualized in real-time.

\section{Conclusions and perspectives}

With continuous research efforts from researchers worldwide, AIEgens have found increasing applications in healthcarerelated areas and contributed to the development of the associated technologies. In this review, we summarized some recent progress on the employment of AIEgens for healthcare-related research. Three aspects of investigations on their applications were reviewed, namely, diagnosis, theragnosis and mechanistic studies and personalized medicines. In the diagnosis section, we introduced the utilization of AIEgens for cytogenetics studies, evaluation of sperm cell activity, detection of CTCs, intraoperative pathological imaging of bone microcracks and tracking of atherosclerotic plaques. In these examples, AIEgens served as fluorescence reporters or indicators to report the presence and quantity of targets, or highlight targets for better differentiation in clinical samples. In the theragnosis part, we elaborated on the applications of AIEgens as both probes and PSs and selected some examples on the theragnosis of bacterial infection, cancer, as well as developing novel approaches to improve the theragnosis outcome, such as elevating spatial precision, minimizing toxicity, and increasing PDT efficiency. These works not only contributed to our comprehensive understanding of theragnosis but also promoted the practical applications of AIEgens. In the third part, we discussed gaining mechanistic insight and providing guidance for personalized medicine. In these works, AIEgens contributed to the understanding of biological processes as well as the innovation of regular experimental operations.

Research on AIEgens for healthcare is advancing at a high speed. We believe more interesting and practically useful AIEgens will be developed and applied in clinical settings. Future developments may be focused on the following areas:

(1) The development of a toolbox of AIEgens for clinical diagnosis. Future research efforts can be spent to increase the analyte variety and build up a toolbox for clinical diagnosis. Probes for varied targets in body liquids, such as blood, urine and semen. When a full set of AIEgen-based diagnosis becomes available, it will greatly promote widespread clinical applications.

(2) Integrating AIEgens into detection kits or devices. Most current research efforts are focused on the development of materials. To step forward, AIEgens should be integrated into ready-to-use diagnosis kits or devices, such as detection kits for enzymes and proteins in blood, portable devices employing 
AIEgens for varied cancer-related biomarkers, and testing strips for antigens. Such ready-to-use products will facilitate the promotion of these techniques to doctors in hospitals. Thus, experts with varied backgrounds, such as mechanics, electronics, etc., should participate in the development.

(3) Research on more clinical testing. In clinics, AIEgens will be working in more complex environments with varied interferences. AIEgens should get rid of these interferences and provide reliable and consistent results before they can be widely used. Thus, collaborations with doctors should be encouraged.

With continuous research efforts and varied expertise in multiple disciplines, the progress of clinical applications of AIEgens will speed up and AIEgens could benefit aspects of human health.

\section{Conflicts of interest}

There are no conflicts to declare.

\section{Acknowledgements}

This work is partially supported by the National Natural Scientific Foundation of China (Grant Number: 22005050). S. Chen acknowledge the support from the startup funding from Ming Wai Lau Centre for Reparative Medicine, Karolinska Institutet, the Innovation and Technology Commission (ITS/022/18, ITC, HK).

\section{Notes and references}

1 G. H. Lee, H. Moon, H. Kim, G. H. Lee, W. Kwon, S. Yoo, D. Myung, S. H. Yun, Z. Bao and S. K. Hahn, Multifunctional materials for implantable and wearable photonic healthcare devices, Nat. Rev. Mater., 2020, 5, 149-165.

2 X. Michalet, Quantum dots for live cells, in vivo imaging, and diagnostics, Science, 2005, 307, 538-544.

3 B. N. G. Giepmans, S. R. Adams, M. H. Ellisman and R. Y. Tsien, The fluorescent toolbox for assessing protein location and function, Science, 2006, 312, 217-224.

4 G. Ulrich, R. Ziessel and A. Harriman, The chemistry of fluorescent bodipy dyes: versatility unsurpassed, Angew. Chem., Int. Ed., 2008, 47, 1184-1201.

5 R. Epple and T. Forster, Quenching and degree of polarization of fluorescence in solutions, Z. Elektrochem. Angew. Phys. Chem., 1954, 58, 783-787.

6 T. Forster and K. Kasper, Concentration reversal of the fluorescence of pyrene, Z. Elektrochem. Angew. Phys. Chem., 1955, 59, 976-980.

7 Y. Hong, J. W. Y. Lam and B. Z. Tang, Aggregation-induced emission, Chem. Soc. Rev., 2011, 40, 5361-5388.

8 J. Mei, Y. Hong, J. W. Y. Lam, A. Qin, Y. Tang and B. Z. Tang, Aggregation-induced emission: the whole is more brilliant than the parts, Adv. Mater., 2014, 26, 5429-5479.

9 J. Luo, Z. Xie, Z. Xie, J. W. Y. Lam, L. Cheng, H. Chen, C. Qiu, H. S. Kwok, X. Zhan, Y. Liu, D. Zhu, B. Z. Tang, B. Z. Tang and B. Z. Tang, Aggregation-induced emission of 1-methyl-1,2,3,4,5pentaphenylsilole, Chem. Commun., 2001, 1740-1741.
10 H. Gao, X. Zhang, C. Chen, K. Li and D. Ding, Unity makes strength: how aggregation-induced emission luminogens advance the biomedical field, Adv. Biosyst., 2018, 2, 1-27.

11 R. T. K. Kwok, C. W. T. Leung, J. W. Y. Lam and B. Z. Tang, Biosensing by luminogens with aggregation-induced emission characteristics, Chem. Soc. Rev., 2015, 44, 4228-4238.

12 E. Zhao, P. Lai, Y. Xu, G. Zhang and S. Chen, Fluorescent materials with aggregation-induced emission characteristics for array-based sensing assay, Front. Chem., 2020, 8, 566.

13 D. Ding, K. Li, B. Liu and B. Z. Tang, Bioprobes based on AIE fluorogens, Acc. Chem. Res., 2013, 46, 2441-2453.

$14 \mathrm{H}$. Gao, X. Zhao and S. Chen, AIEgen-based fluorescent nanomaterials: fabrication and biological applications, Molecules, 2018, 23, 1-20.

15 C. W. T. Leung, Y. Hong, S. Chen, E. Zhao, J. W. Y. Lam and B. Z. Tang, A photostable AIE luminogen for specific mitochondrial imaging and tracking, J. Am. Chem. Soc., 2013, 135, 62-65.

16 X. Gu, E. Zhao, J. W. Y. Lam, Q. Peng, Y. Xie, Y. Zhang, K. S. Wong, H. H. Y. Sung, I. D. Williams and B. Z. Tang, Mitochondrion-specific live-cell bioprobe operated in a fluorescence turn-on manner and a well-designed photoactivatable mechanism, Adv. Mater., 2015, 27, 7093-7100.

17 W. Zhang, Y. Huang, Y. Chen, E. Zhao, Y. Hong, S. Chen, J. W. Y. Lam, Y. Chen, J. Hou and B. Z. Tang, Amphiphilic tetraphenylethene-based pyridinium salt for selective cellmembrane imaging and room-light-induced special reactive oxygen species generation, ACS Appl. Mater. Interfaces, 2019, 11, 10567-10577.

18 H. Shi, N. Zhao, D. Ding, J. Liang, B. Z. Tang and B. Liu, Fluorescent light-up probe with aggregation-induced emission characteristics for in vivo imaging of cell apoptosis, Org. Biomol. Chem., 2013, 11, 7289-7296.

19 Y. Chen, M. Li, Y. Hong, J. W. Y. Lam, Q. Zheng and B. Z. Tang, Dual-modal MRI contrast agent with aggregation-induced emission characteristic for liver specific imaging with long circulation lifetime, ACS Appl. Mater. Interfaces, 2014, 6, 10783-10791.

20 Y. Hong, H. Wang, M. Xue, P. Zhang, W. Liu, S. Chen, R. Zeng, J. Cui, Y. Gao and J. Chen, Rational design of ratiometric and lysosome-targetable AIE dots for imaging endogenous HClO in live cells, Mater. Chem. Front., 2019, 3, 203-208.

21 M. Lin, J. Huang, F. Zeng and S. Wu, A fluorescent probe with aggregation-induced emission for detecting alkaline phosphatase and cell imaging, Chem. - Asian J., 2019, 14, 802-808.

22 Y. Fu, H. Liu, X. Zhu, J. Zeng, Z. Zhao and B. Z. Tang, Efficient aggregation-induced delayed fluorescent materials based on bipolar carrier transport materials for the fabrication of high-performance nondoped OLEDs with very small efficiency roll-off, J. Mater. Chem. C, 2020, 8, 9549-9557.

23 J. Guo, Z. Zhao and B. Z. Tang, Purely organic materials with aggregation-induced delayed fluorescence for efficient nondoped OLEDs, Adv. Opt. Mater., 2018, 6, 1-11.

24 W. Feng, Q. Su, Y. Ma, Z. Džolić, F. Huang, Z. Wang, S. Chen and B. Z. Tang, Tetraphenylbenzosilole: an AIE building 
block for deep-blue emitters with high performance in nondoped spin-coating OLEDs, J. Org. Chem., 2020, 85, 158-167.

25 X. Zhang, Y. Zhang, H. Zhang, Y. Quan, Y. Li, Y. Cheng and S. Ye, High brightness circularly polarized organic lightemitting diodes based on nondoped aggregation-induced emission (AIE)-active chiral binaphthyl emitters, Org. Lett., 2019, 21, 439-443.

26 X. Cai, N. Xie, Y. Li, J. W. Y. Lam, J. Liu, W. He, J. Wang and B. Z. Tang, A smart AIEgen-functionalized surface with reversible modulation of fluorescence and wettability, Mater. Horiz., 2019, 6, 2032-2039.

27 W. Luo and G. Wang, Photo-responsive fluorescent materials with aggregation-induced emission characteristics, Adv. Opt. Mater., 2020, 2001362, 1-18.

28 Q. Zhao, X. Y. Dai, H. Yao, Y. M. Zhang, W. J. Qu, Q. Lin and T. B. Wei, Stimuli-responsive supramolecular hydrogel with white AIE effect for ultrasensitive detection of $\mathrm{Fe}^{3+}$ and as rewritable fluorescent materials, Dyes Pigm., 2021, 184, 108875.

29 P. Shi, X. Zhang, Y. Liu, Y. Duan, Y. Li, Z. Li and T. Han, A multi-stimuli-responsive AIE material switching among three emission states, Mater. Lett., 2020, 263, 127214.

30 J. Dong, P. Shen, S. Ying, Z. J. Li, Y. Di Yuan, Y. Wang, X. Zheng, S. B. Peh, H. Yuan, G. Liu, Y. Cheng, Y. Pan, L. Shi, J. Zhang, D. Yuan, B. Liu, Z. Zhao, B. Z. Tang and D. Zhao, Aggregation-induced emission-responsive metal-organic frameworks, Chem. Mater., 2020, 32, 6706-6720.

31 E. Zhao, H. Deng, S. Chen, Y. Hong, C. W. T. Leung, J. W. Y. Lam and B. Z. Tang, A dual functional AEE fluorogen as a mitochondrial-specific bioprobe and an effective photosensitizer for photodynamic therapy, Chem. Commun., 2014, 50, 14451-14454.

32 C. Gui, E. Zhao, R. T. K. Kwok, A. C. S. Leung, J. W. Y. Lam, M. Jiang, H. Deng, Y. Cai, W. Zhang, H. Su and B. Z. Tang, AIE-active theranostic system: selective staining and killing of cancer cells, Chem. Sci., 2017, 8, 1822-1830.

33 Y. Yuan, G. Feng, W. Qin, B. Z. Tang and B. Liu, Targeted and image-guided photodynamic cancer therapy based on organic nanoparticles with aggregation-induced emission characteristics, Chem. Commun., 2014, 50, 8757-8760.

34 F. Hu, S. Xu and B. Liu, Photosensitizers with aggregationinduced emission: materials and biomedical applications, Adv. Mater., 2018, 30, 1801350.

35 C. Zhu, R. T. K. Kwok, J. W. Y. Lam and B. Z. Tang, Aggregation-induced emission: a trailblazing journey to the field of biomedicine, ACS Appl. Bio Mater., 2018, 1, 1768-1786.

36 J. Qian and B. Z. Tang, AIE luminogens for bioimaging and theranostics: from organelles to animals, Chem, 2017, 3, 56-91.

37 M. Gao and B. Z. Tang, AIE-based cancer theranostics, Coord. Chem. Rev., 2020, 402, 213076.

38 X. Gu, R. T. K. Kwok, J. W. Y. Lam and B. Z. Tang, AIEgens for biological process monitoring and disease theranostics, Biomaterials, 2017, 146, 115-135.

39 X. He, L. H. Xiong, Z. Zhao, Z. Wang, L. Luo, J. W. Y. Lam, R. T. K. Kwok and B. Z. Tang, AIE-based theranostic systems for detection and killing of pathogens, Theranostics, 2019, 9, 3223-3248.

40 R. Ideta, F. Tasaka, W. D. Jang, N. Nishiyama, G. D. Zhang, A. Harada, Y. Yanagi, Y. Tamaki, T. Aida and K. Kataoka, Nanotechnology-based photodynamic therapy for neovascular disease using a supramolecular nanocarrier loaded with a dendritic photosensitizer, Nano Lett., 2005, 5, 2426-2431.

41 W.-D. Jang, N. Nishiyama, G.-D. Zhang, A. Harada, D.-L. Jiang, S. Kawauchi, Y. Morimoto, M. Kikuchi, H. Koyama, T. Aida and K. Kataoka, Supramolecular nanocarrier of anionic dendrimer porphyrins with cationic block copolymers modified with polyethylene glycol to enhance intracellular photodynamic efficacy, Angew. Chem., 2005, 117, 423-427.

42 N. Nishiyama, W. D. Jang and K. Kataoka, Supramolecular nanocarriers integrated with dendrimers encapsulating photosensitizers for effective photodynamic therapy and photochemical gene delivery, New J. Chem., 2007, 31, 1074-1082.

43 M. A. Oar, J. M. Serin, W. R. Dichtel, J. M. J. Fréchet, T. Y. Ohulchanskyy and P. N. Prasad, Photosensitization of singlet oxygen via two-photon-excited fluorescence resonance energy transfer in a water-soluble dendrimer, Chem. Mater., 2005, 17, 2267-2275.

44 B. Gao, Y. Liu, H. Yin, Y. Li, Q. Bai and L. Zhang, Water-soluble dendritic polyaspartic porphyrins: potential photosensitizers for photodynamic therapy, New J. Chem., 2012, 36, 28-31.

45 K. Liu, Y. Liu, Y. Yao, H. Yuan, S. Wang, Z. Wang and X. Zhang, Supramolecular photosensitizers with enhanced antibacterial efficiency, Angew. Chem., Int. Ed., 2013, 52, 8285-8289.

46 F. Hu, S. Xu and B. Liu, Photosensitizers with aggregationinduced emission: materials and biomedical applications, Adv. Mater., 2018, 30, 1801350.

47 G. Feng, G. Q. Zhang and D. Ding, Design of superior phototheranostic agents guided by Jablonski diagrams, Chem. Soc. Rev., 2020, 49, 8179-8234.

48 L. Qian, Q. Li, K. Baryeh, W. Qiu, K. Li, J. Zhang, Q. Yu, D. Xu, W. Liu, R. E. Brand, X. Zhang, W. Chen and G. Liu, Biosensors for early diagnosis of pancreatic cancer: a review, Transl. Res., 2019, 213, 67-89.

49 Y. Lyu, D. Cui, J. Huang, W. Fan, Y. Miao and K. Pu, Nearinfrared afterglow semiconducting nano-polycomplexes for the multiplex differentiation of cancer exosomes, Angew. Chem., Int. Ed., 2019, 58, 4983-4987.

50 E. Pergament, The future of prenatal diagnosis and screening, J. Clin. Med, 2014, 3, 1291-1301.

51 M. Y. Wu, J. K. Leung, L. Liu, C. Kam, K. Y. K. Chan, R. A. Li, S. Feng and S. Chen, A small-molecule AIE chromosome periphery probe for cytogenetic studies, Angew. Chem., Int. Ed., 2020, 59, 10327-10331.

52 Z. Zeng, X. Ren, T. Yin, X. Gao, M. Tsai, Y. Zhang and M. Gu, Multiplexed detection and the establishment of a novel high-throughput method for human germ cell quality screening based on aggregation-induced emission, Am. J. Transl. Res., 2019, 11, 6907-6923.

53 N. Zhao, S. Chen, Y. Hong and B. Z. Tang, A red emitting mitochondria-targeted AIE probe as an indicator for 
membrane potential and mouse sperm activity, Chem. Commun., 2015, 51, 13599-13602.

54 J. G. Lohr, V. A. Adalsteinsson, K. Cibulskis, A. D. Choudhury, M. Rosenberg, P. Cruz-Gordillo, J. M. Francis, C. Z. Zhang, A. K. Shalek, R. Satija, J. J. Trombetta, D. Lu, N. Tallapragada, N. Tahirova, S. Kim, B. Blumenstiel, C. Sougnez, A. Lowe, B. Wong, D. Auclair, E. M. Van Allen, M. Nakabayashi, R. T. Lis, G. S. M. Lee, T. Li, M. S. Chabot, A. Ly, M. E. Taplin, T. E. Clancy, M. Loda, A. Regev, M. Meyerson, W. C. Hahn, P. W. Kantoff, T. R. Golub, G. Getz, J. S. Boehm and J. C. Love, Whole-exome sequencing of circulating tumor cells provides a window into metastatic prostate cancer, Nat. Biotechnol., 2014, 32, 479-484.

55 B. Situ, X. Ye, Q. Zhao, L. Mai, Y. Huang, S. Wang, J. Chen, B. Li, B. He, Y. Zhang, J. Zou, B. Z. Tang, X. Pan and L. Zheng, Identification and single-cell analysis of viable circulating tumor cells by a mitochondrion-specific AIE bioprobe, Adv. Sci., 2020, 7, 1902760.

56 J. Zhang, Y. Song, F. Xia, C. Zhu, Y. Zhang, W. Song, J. Xu and $\mathrm{X}$. Ma, Rapid and accurate intraoperative pathological diagnosis by artificial intelligence with deep learning technology, Med. Hypotheses, 2017, 107, 98-99.

57 Z. Kaufman, S. Lew, B. Griffel and A. Dinbar, Frozen-section diagnosis in surgical pathology: a prospective analysis of 526 frozen sections, Cancer, 1986, 57, 377-379.

58 D. Chen, H. Mao, Y. Hong, Y. Tang, Y. Zhang, M. Li and Y. Dong, Hexaphenyl-1,3-butadiene derivative: A novel 'turnon' rapid fluorescent probe for intraoperative pathological diagnosis of hepatocellular carcinoma, Mater. Chem. Front., 2020, 4, 2716-2722.

59 E. A. Zimmermann and R. O. Ritchie, Bone as a structural material, Adv. Healthcare Mater., 2015, 4, 1287-1304.

60 M. Gao, Y. Li, X. Chen, S. Li, L. Ren and B. Z. Tang, Aggregation-induced emission probe for light-up and in situ detection of calcium Ions at high concentration, ACS Appl. Mater. Interfaces, 2018, 10, 14410-14417.

61 B. Situ, M. Gao, X. He, S. Li, B. He, F. Guo, C. Kang, S. Liu, L. Yang, M. Jiang, Y. Hu, B. Z. Tang and L. Zheng, A twophoton AIEgen for simultaneous dual-color imaging of atherosclerotic plaques, Mater. Horiz., 2019, 6, 546-553.

62 N. Song, Z. Zhang, P. Liu, Y. W. Yang, L. Wang, D. Wang and B. Z. Tang, Nanomaterials with supramolecular assembly based on AIE luminogens for theranostic applications, Adv. Mater., 2020, 32, 2004208.

63 X. Cai and B. Liu, Aggregation-induced emission: Recent advances in materials and biomedical applications, Angew. Chem., Int. Ed., 2020, 59, 9868-9886.

64 J. Dai, X. Wu, S. Ding, X. Lou, F. Xia, S. Wang and Y. Hong, Aggregation-induced emission photosensitizers: from molecular design to photodynamic therapy, J. Med. Chem., 2020, 63, 1996-2012.

65 C. Wang, X. Zhao, H. Jiang, J. Wang, W. Zhong, K. Xue and C. Zhu, Transporting mitochondrion-targeting photosensitizers into cancer cells by low-density lipoproteins for fluorescence-feedback photodynamic therapy, Nanoscale, 2021, 13, 1195-1205.
66 M. Kang, Z. Zhang, N. Song, M. Li, P. Sun, X. Chen, D. Wang and B. Z. Tang, Aggregation-enhanced theranostics: AIE sparkles in biomedical field, Aggregate, 2020, 1, 80-106.

67 Y. Zhang, X. Zhao, Y. Li, X. Wang, Q. Wang, H. Lu and L. Zhu, A fluorescent photosensitizer with far red/near-infrared aggregation-induced emission for imaging and photodynamic killing of bacteria, Dyes Pigm., 2019, 165, 53-57.

68 T. Zhou, R. Hu, L. Wang, Y. Qiu, G. Zhang, Q. Deng, H. Zhang, P. Yin, B. Situ, C. Zhan, A. Qin and B. Z. Tang, An AIE-active conjugated polymer with high ROS-generation ability and biocompatibility for efficient photodynamic therapy of bacterial infections, Angew. Chem., Int. Ed., 2020, 59, 9952-9956.

69 N. Alifu, X. Dong, D. Li, X. Sun, A. Zebibula, D. Zhang, G. Zhang and J. Qian, Aggregation-induced emission nanoparticles as photosensitizer for two-photon photodynamic therapy, Mater. Chem. Front., 2017, 1, 1746-1753.

70 L. Zhang, W. Che, Z. Yang, X. Liu, S. Liu, Z. Xie, D. Zhu, Z. Su, B. Z. Tang and M. R. Bryce, Bright red aggregationinduced emission nanoparticles for multifunctional applications in cancer therapy, Chem. Sci., 2020, 11, 2369-2374.

71 X. He, B. Situ, M. Gao, S. Guan, B. He, X. Ge, S. Li, M. Tao, H. Zou, B. Z. Tang and L. Zheng, Stereotactic photodynamic therapy using a two-photon AIE photosensitizer, Small, 2019, 15, 1905080.

72 Y. Yang, L. Wang, H. Cao, Q. Li, Y. Li, M. Han, H. Wang and J. Li, Photodynamic therapy with liposomes encapsulating photosensitizers with aggregation-induced emission, Nano Lett., 2019, 19, 1821-1826.

73 X. Gu, X. Zhang, H. Ma, S. Jia, P. Zhang, Y. Zhao, Q. Liu, J. Wang, X. Zheng, J. W. Y. Lam, D. Ding and B. Z. Tang, Corannuleneincorporated AIE nanodots with highly suppressed nonradiative decay for boosted cancer phototheranostics in vivo, Adv. Mater., 2018, 30, 1801065.

74 J. Huang, B. He, Z. Zhang, Y. Li, M. Kang, Y. Wang, K. Li, D. Wang and B. Z. Tang, Aggregation-induced emission luminogens married to 2D black phosphorus nanosheets for highly efficient multimodal theranostics, Adv. Mater., 2020, 32, 2003382.

75 Z. Zhang, W. Xu, M. Kang, H. Wen, H. Guo, P. Zhang, L. Xi, K. Li, L. Wang, D. Wang and B. Z. Tang, An all-round athlete on the track of phototheranostics: subtly regulating the balance between radiative and nonradiative decays for multimodal imaging-guided synergistic therapy, $A d v$. Mater., 2020, 32, 2003210.

76 C. Chen, X. Ni, S. Jia, Y. Liang, X. Wu, D. Kong and D. Ding, Massively evoking immunogenic cell death by focused mitochondrial oxidative stress using an AIE luminogen with a twisted molecular structure, Adv. Mater., 2019, 31, 1904914.

77 J. Wang, J. Li, L. Wang, T. Han, D. Wang and B. Z. Tang, AIEgen-based polymer nanocomposites for imaging-guided photothermal therapy, ACS Appl. Polym. Mater., 2020, 2, 4306-4318.

78 J. Chen, M. Gao, L. Wang, S. Li, J. He, A. Qin, L. Ren, Y. Wang and B. Z. Tang, Aggregation-induced emission probe for study of the bactericidal mechanism of antimicrobial peptides, ACS Appl. Mater. Interfaces, 2018, 10, 11436-11442. 
79 X. Shi, S. H. P. Sung, J. H. C. Chau, Y. Li, Z. Liu, R. T. K. Kwok, J. Liu, P. Xiao, J. Zhang, B. Liu, J. W. Y. Lam and B. Z. Tang, Killing $\mathrm{G}(+)$ or $\mathrm{G}(-)$ bacteria? The important role of molecular charge in AIE-active photosensitizers, Small Methods, 2020, 4, 2000046.

80 E. Zhao, Y. Chen, S. Chen, H. Deng, C. Gui, C. W. T. Leung, Y. Hong, J. W. Y. Lam and B. Z. Tang, A luminogen with aggregation-induced emission characteristics for wash-free bacterial imaging, high-throughput antibiotics screening and bacterial susceptibility evaluation, Adv. Mater., 2015, 27, 4931-4937.

$81 \mathrm{M}$. H. Yun, Changes in regenerative capacity through lifespan, Int. J. Mol. Sci., 2015, 16, 25392-25432.
82 X. Shi, N. Yan, G. Niu, S. H. P. Sung, Z. Liu, J. Liu, R. T. K. Kwok, J. W. Y. Lam, W. X. Wang, H. H. Y. Sung, I. D. Williams and B. Z. Tang, In vivo monitoring of tissue regeneration using a ratiometric lysosomal AIE probe, Chem. Sci., 2020, 11, 3152-3163.

$83 \mathrm{~J} . \mathrm{K}$. Biehl and B. Russell, Introduction to stem cell therapy, J. Cardiovasc. Nurs., 2009, 24, 98-103.

84 X. Cai, C. J. Zhang, F. Ting Wei Lim, S. J. Chan, A. Bandla, C. K. Chuan, F. Hu, S. Xu, N. V. Thakor, L. De Liao and B. Liu, Organic nanoparticles with aggregation-induced emission for bone marrow stromal cell tracking in a rat PTI model, Small, 2016, 12, 6576-6585. 